\title{
RECURSOS ECONÓMICOS Y CALIDAD DE VIDA EN LA POBLACIÓN MAYOR
}

\section{ECONOMIC RESOURCES AND QUALITY OF LIFE AMONG OLD PEOPLE}

\author{
Vicente Rodríguez-Rodríguez, Fermina Rojo-Pérez, Gloria Fernández-Mayoralas \\ y Karim Ahmed Mohamed \\ Instituto de Economía y Geografía. Centro de Ciencias Humanas y Sociales (CSIC). Madrid. España
}

RAÚL LARDIÉS-BOSQUE

Departamento de Geografía y Ordenación del Territorio. Universidad de Zaragoza. España

\author{
María-Eugenia Prieto-Flores \\ Instituto de Salud Carlos III. Madrid. España
}

José Manuel Rojo-Abuín

Unidad de Análisis Estadístico en el CCHS (CSIC). Madrid. España

vicente.rodriguez@cchs.csic.es; fermina.rojo@cchs.csic.es; gloria.fernandezmayoralas@cchs.csic.es; karim.ahmed@cchs.csic.es; rlardies@unizar.es; mprieto@isciii.es; josemanuel.rojo@cchs.csic.es

\section{RESUMEN \\ Una de las dimensiones que definen la Calidad de Vida de los mayores son los recursos económicos. A partir de la encuesta CadeViMa se han utilizado diversos indicadores para definir grupos de individuos significados por su valoración objetiva y subjetiva de los recursos económicos: los pesimistas sin base económica (privación) y con base económica (disonancia), y los optimistas sin base económica (adaptación) y con base económica (bienestar), de acuerdo con la teoría de las condiciones de vida y el bienestar subjetivo. La situación económica de los mayores y su valoración están fundamentadas en sus condiciones personales y en otras facetas de su bienestar, habiéndose comprobado que, entre ellas, la edad, el nivel educativo, el estado de salud, las redes familiar y social y la participación en determinadas actividades de ocio son las que presentan relaciones más significativas con la dimensión económica en el contexto de su calidad de vida.}

\section{Palabras Clave}

Bienestar, Dimensión económica, Envejecimiento, España, Indicadores objetivos y subjetivos, Madrid, Satisfacción con la vida.

\section{Abstract}

Economic resources are an important domain in Quality of Life (QoL) in later ages. Several characteristics, taken from the CadeViMa survey, are used to cluster old people, based on the objective and economic resources and their subjective assessments. The groups depicted were the pessimists without or with enough economic resources (deprivation, dissonance), and the optimists without and with economic base (adaptation and well-being) according to the theory of objective living conditions and subjective well-being. Elderly personal features and other factors were analysed to ground their economic assessment and the self-economic evaluation, arising the age, health status, social and family networks and leisure activities as the most significant explaining factors.

\section{KEYWORDS}

Ageing, Economic Domain, Life Satisfaction, Objective and Subjective Indicators, Madrid, Spain, Well-being. 


\section{INTRODUCCIÓN: ESTADO ACTUAL DEL TEMA Y OBJETIVOS DE ESTUDIO ${ }^{1}$}

Los recursos económicos influyen en los comportamientos sociales y en la Calidad de Vida (CdV) de la población, especialmente de las personas mayores, y tienden a formar parte de muchos estudios sociales y económicos sobre esta poblacion (Sánchez Vera 2000). Es habitual que la "renta económica" sea usada como medida del bienestar de los Estados, además de otros conceptos similares (riqueza, transferencias de capital, ahorros, etc.) con el objetivo de comparar grupos de población y países (Gornick et al. 2009). Cuando se analizan los individuos, el debate sobre las medidas mejor adaptadas a su análisis y sobre su influencia en la CdV a través de diversas perspectivas (Walker 2005) se amplía para referirse a la educación, la ocupación, la renta monetaria o la riqueza patrimonial como posibles factores condicionantes. De acuerdo con esta posición teórica, la hipótesis de partida es que la capacidad/incapacidad de la persona para participar en las actividades sociales y en la vida de la comunidad está en función de su nivel económico, generalmente medido a partir de los recursos económicos, valorados objetiva y subjetivamente.

Con un carácter general, los recursos económicos de los mayores suelen servir para la previsión futura de la cobertura económica por parte de la Seguridad Social, en beneficio de las pensiones privadas (Lassey y Lassey 2001; Pérez Ortiz 2006), para constatar la importancia de las rentas públicas y privadas, para valorar las transferencias en el ámbito familiar, especialmente cuando las redes familiares y sociales tienen una gran importancia como factor de influencia en la CdV de los mayores (Polverini y Lamura 2005), o para prever los riesgos de caer en la pobreza según el sexo o la forma de convivencia (Pérez Ortiz 1997; Sánchez Vera 2000; Walker y Hennesy 2004). También tiene interés la posición del crecimiento económico como condición para el incremento de la CdV con un carácter poblacional, relación no siempre demostrada a escala individual (Diener 1984; Schyns 1998).

Sin embargo y como diversas investigaciones han señalado, los estudios sobre los recursos económicos de las personas mayores en España tienden a orientarse hacia otras visiones. Así, entre las posibilidades que tienen campos de mayor desarrollo científico podrían destacarse la emergencia de poblaciones jubiladas para las que la independencia y el control económico de su vida son factores determinantes de la toma de decisiones (Trinidad 2008), la valoración de estrategias de seguridad económica para el

\footnotetext{
${ }^{1}$ Los autores agradecen los útiles comentarios de los evaluadores anónimos que han contribuido a mejorar la versión inicial presentada. La investigación que sirve de base a este artículo ha sido financiada con cargo a dos proyectos: i) Calidad de Vida de los mayores no institucionalizados en España. Una contribución al estudio de la Calidad de Vida desde una aproximación holística. MCYT, Plan Nacional I+D+I 20002003, Programa Nacional de Promoción General del Conocimiento, Ref. BSO2003-00401. ii) Evaluación de la Calidad de Vida de la población mayor en la Comunidad de Madrid. Dirección General de Investigación, Consejería de Educación, Comunidad de Madrid, Ref. 06/HSE/0417/2004.
} 
futuro basada en las diversas formas de ahorro y usos del patrimonio económico (Sanchez Vera 2000; Trinidad 2008), u otros aspectos económicos de interés actual y futuro como el gasto y el consumo (Pérez Ortiz 1997; Bazo 1999; Sánchez Vera 2000) y el uso del tiempo y su valor económico (Sánchez Vera 2000; Durán y Rogero 2009).

En cuanto a la perspectiva individual, la dimensión económica aparece tradicionalmente como constitutiva de la CdV, junto a otras dimensiones básicas (Smith 2000; Fernández-Mayoralas y Rojo 2005), ya que son las circunstancias personales, generacionales y de organización social, cualquiera que sea la medida que de ellas se adopte, las que influyen de una manera decisiva sobre el bienestar (Pérez Ortiz 1997). En los estudios sobre población mayor, los "recursos económicos" están relacionados con la capacidad del individuo para proveer sus necesidades básicas (Scharf et al. 2000), mostrando una asociación directa entre cantidad de recursos (como medida objetiva) y CdV (Sirgy 2001) y entre la trayectoria vital de los individuos y la tenencia y disfrute de recursos en la vejez. Ello podría influir en la evaluación subjetiva de la realidad (Nguyen et al. 2008), específicamente en la salud (Berry 2007) y en el proceso de reducción de las desigualdades de salud en países como España (Regidor et al. 2006) en comparación con otros europeos (Mackenback 2006). Sin embargo, ciertos mecanismos psicológicos permiten destacar discrepancias entre lo que uno tiene, como recurso económico, lo que desea 0 calcula que debería de tener para cubrir mejor sus necesidades personales, y lo que otros tienen como marco de comparación interpersonal; y ello condiciona la forma de comportarse y las opiniones emitidas sobre la CdV que se disfruta en relación con los recursos económicos (Michalos 2003). Además, la adecuación entre recursos y necesidades, como indicador de CdV, tiene un significado distinto de acuerdo con los rasgos sociodemográficos individuales (Lamura 2003). Con un carácter prospectivo, las encuestas generales sobre valoración de la situación general de una sociedad permiten apreciar cómo la percepción de los problemas económicos en relación con el sentimiento de seguridad de los individuos está condicionada por los rasgos sociodemográficos de los individuos ${ }^{2}$.

Otra perspectiva es el debate sobre el papel de los indicadores objetivos (sociales, normativos que miden las diferencias en los niveles de bienestar general o personal) y subjetivos (personales) en la medida de la CdV (Friedman 1997; Lamura et al. 2003). En este ámbito es habitual dotar a esos indicadores de un carácter complementario, con ventajas y dificultades, dada la conveniencia de realizar una valoración amplia de los fenómenos sociales en entornos individuales, sociales y culturales cambiantes (Diener

\footnotetext{
${ }^{2}$ Así, y en relación con la emergencia de la crisis económica en 2008, el Centro de Investigaciones Sociológicas (CIS) incluyó en el Barómetro 2801 de mayo de 2009 (http://www.cis.es/cis/opencm/ES/1_encuestas/ estudios/ver.jsp?estudio $=9400$ ) una valoración de los problemas generales y personales de más importancia para los entrevistados, destacando en ese momento el de la 'situación económica' $(40 \%)$, menos valorado entre los mayores de 65 años (29\%) y los de menor nivel educativo (33\%) que entre los menores de $35(44 \%)$ y los encuestados con estudios superiores (42\%).
} 
y Suh 1997). De lo que quedan pocas dudas es de la relación entre la percepción de la situación social y los recursos económicos y la evaluación subjetiva del estado de salud (Demakakos et al. 2008).

A partir de la bibliografía analizada y de la reflexión recogida en el estudio de Brown et al. (2004) se comprueba de forma clara la importancia de la situación económica de las personas mayores como dominio fundamental de su CdV. En este contexto, los objetivos de este trabajo son tres. El primero pretende un acercamiento general a las condiciones y la calidad de vida de los mayores relacionadas con sus recursos económicos, para conocer cuál es su "significado presente y futuro" según las necesidades actuales y previstas, para examinar la "sensación o el sentimiento de seguridad" que producen los recursos económicos (el capital acumulado durante su etapa laboral) en las personas, durante los últimos años de su vida (Walker y Hennessy 2004), cuando se tienen ya comportamientos orientados al ahorro 0 al consumo, y para valorar la "satisfacción" con los recursos por parte de quien, previsiblemente, puede encontrarse en una situación de medios económicos escasos porque no ha tenido posibilidades de conseguirlos, pero que desconoce otros escenarios (Smith 2000). El segundo objetivo persigue definir una "tipología" de población mayor de acuerdo con sus recursos económicos, medidos objetiva y subjetivamente, en línea con la teoría de la CdV basada en condiciones objetivas de vida y bienestar subjetivo (Noll 2002). Finalmente, como tercer objetivo, se evaluará el papel que juegan los rasgos sociodemográficos y el entorno físico y social como "factores condicionantes" de la valoración del balance entre recursos disponibles, oportunidades y necesidades del individuo mayor. De cómo resulte ese balance se derivará una situación vital condicionada por la mayor o menor autonomía personal, especialmente en el caso de las mujeres mayores (Bryant et al. 2002).

\section{Fuentes y MetodologíA: ¿Cómo MediR LOS HeCHOS ECONÓMICOS?}

Son variados los conceptos (ingresos, rentas, independencia financiera, propiedad de la vivienda como elemento patrimonial,...) y las formas de medirlos que se describen en la literatura científica, puestos en conexión con otros componentes relacionados como la satisfacción con el entorno urbano, la seguridad física en la vida, o las condiciones materiales de la vivienda. Por ello, el análisis del valor real de los recursos económicos y sus significados en la CdV de los mayores, a partir de la complementariedad de datos objetivos y subjetivos, ha seguido en este trabajo los estándares habituales (Sirgy 2001).

Para los propósitos de este artículo se ha utilizado la encuesta Calidad de Vida a los Mayores en Madrid (CadeViMa), realizada en el año 2005 a 499 personas, como muestra significativa de la población de 65 y más años residente en vivienda familiar en la Comunidad de Madrid, bajo un muestreo aleatorio estratificado con afijación proporcional al tamaño de los estratos (sexo, edad, y tamaño del hábitat de residencia). El error permitido fue $<4,4 \%$ para una probabilidad de 0,05 y un intervalo de confianza del 
$95 \%$. El cuestionario semi-estructurado contiene preguntas sobre CdV global y dominio especifica; además, se incluyó el Schedule for the Evaluation of Individual Quality of Life-Direct Weighting (SEIQoL-DW), como instrumento de medida de la CdV Individual (Browne et al. 1997; Hickey et al. 1999). Se trata de una encuesta de temática amplia con el propósito de estudiar la multidimensionalidad de la CdV bajo el doble enfoque objetivo y subjetivo. Para la dimensión "recursos económicos" no se solicita una información excesiva con el fin de evitar dos dificultades en su recogida: la sensibilidad que producen las cuestiones económicas, especialmente entre la población mayor, y el notable esfuerzo de recuerdo requerido, ambos aspectos contraproducentes para la calidad de los datos obtenidos y la finalidad de la encuesta. En definitiva, se perseguía conseguir una tasa de respuesta más alta.

En el contexto particular de los recursos económicos, se tomaron datos objetivos y subjetivos sobre cinco cuestiones. Dos de ellas pretenden un acercamiento al conocimiento objetivo de los ingresos totales mensuales recibidos; la primera es el origen de los recursos económicos, con el propósito de identificar la mayor o menor dependencia de los ingresos de una fuente única o múltiple, y las consecuencias que de ello pueden derivarse para la forma de vida de los mayores, bajo la hipótesis de que la mayoría de los encuestados podrían obtenerlos exclusivamente de una pensión. La pregunta se diseñó en formato "multi-respuesta" de manera que cualquiera de las respuestas pudiera entrar en combinación con el resto, atendiendo a la mayor o menor ocurrencia para definir una tipología de individuos. La segunda cuestión es el nivel de ingresos, en una escala ordinal de seis intervalos, suficientemente amplia para aminorar el recelo o las dificultades para responder.

Desde un punto de vista subjetivo, se plantearon tres cuestiones. En primer lugar, hay dos preguntas de tipo perceptual: una, sobre satisfacción con los recursos económicos (considerando ingresos económicos mensuales, ahorros y otros tipos de renta, así como otras pertenencias), en su papel de "facilitadores" de un bienestar personal y familiar; $y$ otra, sobre el sentimiento de seguridad hacia su futuro económico. Como contraste de la información objetiva de ingresos, también se ha recabado el auto-posicionamiento económico del "hogar" del individuo en una escala de 1 a 10, desde la posición "baja" a "alta".

El estudio de la dimensión económica de los mayores se ha cotejado con determinados aspectos relacionados con otras dimensiones de la CdV también obtenidos de la encuesta CadeViMa, con objeto de valorar las circunstancias que pueden estar condicionando este dominio. Su elección fue una tarea ardua porque implicaba tener presentes aspectos que, hipotéticamente, tendrían relación con la dimensión económica, pero que no siempre es posible documentar su asociación significativa. Por ello, una vez hecha una primera revisión de la información disponible, se consideró adecuado tomar en cuenta 6 bloques informativos, relacionados con las características personales y las cinco dimensiones de CdV más nominadas (Fernández-Mayoralas et al. 2007) y con el escenario de vida: (i) los rasgos sociodemográficos de los entrevistados -sexo, edad, nivel de estudios, clase social obtenida de la combinación del nivel de instrucción y la situación socioeconómica (Rojo-Pérez et al. 2007)-, (ii) el estado de salud y 
funcionamiento (Fernández-Mayoralas et al. 2007), (iii) el hogar y la forma de convivencia (Ahmed-Mohamed et al. 2009), (iv) funcionamiento/dependencia y redes de apoyo (Rojo-Pérez et al. 2009a; 2009b), (v) las actividades de ocio y tiempo libre (LardiésBosque et al. 2007), y (vi) el entorno residencial (Rojo-Pérez et al. 2007).

Las variables independientes utilizadas han sido, primero, cruzadas con aquéllas seleccionadas relativas a los recursos económicos, con el fin de validar la representatividad de la relación mediante el test de la $\chi^{2}$ con $p$-values $\leq 0,05$, para, luego, desarrollar un análisis estadístico por cada bloque temático utilizando diversos instrumentos estadísticos y gráficos. Se han empleado técnicas orientadas a reducir la información identificando variables latentes y no directamente medidas (Análisis Factorial), a agrupar individuos con comportamientos semejantes en grupos homogéneos (Análisis Clúster) y a identificar asociación de categorías de variables (Análisis de Árboles Jerárquicos), aplicando los módulos correspondientes de SPPS versión 17 y Answer Tree versión 3.1.

\section{Los recursos económicos en el contexto de la Calidad de Vida de los mayores}

Estudios previos han demostrado que, para los mayores residentes en vivienda familiar en la Comunidad de Madrid, la dimensión "situación económica" ocupa el tercer lugar de entre los 5 dominios de vida más mencionados; detrás de la salud y la red familiar -el $76 \%, 96 \%$ y $80 \%$, respectivamente, de los mayores nominan dichas dimensiones (Fernández-Mayoralas et al. 2007)-. Sin embargo, en el contexto del nivel de satisfacción con cada una de las dimensiones, en una escala de 0 a 100, la situación económica pasa a ocupar el cuarto lugar (valor 60 ), detrás de la red familiar, la salud y la red social (con valores de 77,71 y 66 , respectivamente), y seguida muy de cerca por el componente ocio y tiempo libre con un nivel de satisfacción de 59 .

En general, hablar de la situación económica de la población anciana en España es poner de manifiesto algunos datos que dejan entrever, primero, la desigual valoración de los recursos económicos cuando se hace a escala individual o de hogar, en relación con la fuente de datos y el método de análisis (Pérez Ortiz 1997; Alfageme 1999); y, segundo, su posición desventajosa con respecto a otros grupos de población e, internamente a este conjunto, de unos colectivos (mujeres, viudas, personas que viven en soledad) en relación con otros (Pérez Ortiz 1997; Instituto de Mayores y Servicios Sociales 2007). Así, una buena parte de este grupo demográfico $(80 \%)$ recibe pensiones contributivas de la Seguridad Social con un valor medio, a junio de 2006, de 722 euros mensuales (876 euros en Madrid) (Instituto de Mayores y Servicios Sociales 2007), y con evidentes desequilibrios entre conjuntos de perceptores, con valores más bajos para las pensiones de viudedad y no contributivas, esto es, para los más mayores y las mujeres, todo ello en relación con la actividad laboral previa del individuo.

La población anciana en Madrid no parece alejarse de ese modelo distributivo. Aproximadamente, una tercera parte de los encuestados no respondió a la pregunta sobre los "ingresos totales mensuales percibidos", en la línea de otras investigaciones 
sociales ${ }^{3}$ y teniendo en cuenta la sensibilidad acerca de este tema entre los mayores que, probablemente, encuentran más reparos que otros grupos de población a informar sobre sus datos económicos, en especial aquéllos con mayor nivel de estudios 0 , consecuentemente, una clase social más alta, puesto que en torno a un $60 \%$ de estos individuos no respondieron a esta cuestión. En este contexto, el nivel económico de los mayores en Madrid en 2005 es bajo, siendo así que, entre quienes respondieron a la pregunta, casi la mitad perciben menos de 600 euros mensuales, y de ellos un $8 \%$ no llega a los 300 euros; por su lado, poco más de un tercio $(34,3 \%)$ declara ingresos entre 601 euros y 900 euros, existiendo un discreto grupo de personas (17\%) cuyos ingresos se sitúan en valores superiores a 901 euros, de modo que el valor medio no alcanza los 600 euros. Como tendencia más probable en el futuro, es previsible un aumento de los recursos económicos disponibles a medida que se vayan incorporando nuevas generaciones de mayores, con una trayectoria laboral basada en actividades económicas terciarias, generadoras de una base económica más consistente (Pérez Ortiz 1997; Alfageme 2008). En este marco, y para la muestra representativa analizada (tabla 1), no se ha observado significación estadística entre el nivel de ingresos y el sexo, porque se entiende que éste está asociado con otras variables explicativas, especialmente el nivel educativo que asume la representación del género en esquemas interpretativos multivariables (Alfageme 1999; Grundy y Holt 2001; Park et al. 2010). Se mantiene, no obstante, la tendencia de un mayor nivel entre los hombres, con respecto a las mujeres, de acuerdo con la disponibilidad de recursos económicos como consecuencia de una trayectoria vital y laboral diferente, con un claro efecto generacional (Pérez Ortiz 1997). Pero también hay otras evidencias que demuestran que la mujer tiende a tener percepciones más negativas con respecto a la situación económica actual y futura (Sánchez Vera 2000), corroboradas en distintas encuestas recientes, probablemente relacionadas con su posición dependiente del hombre en la toma de decisiones económicas familiares de carácter general y otros factores generacionales como la educación (Pérez Ortiz 2006; Abellán et al. 2007).

${ }^{3}$ En la Encuesta Nacional de Salud de 2003 la tasa de no respuesta para la pregunta sobre ingresos fue del 27\% (http://www.msc.es/estadEstudios/estadisticas/encuestaNacional/encuestaNac2003/home.htm) Consultado el 19 de noviembre de 2010. Algo menos, el 19\%, se contabiliza en la Encuesta de Condiciones de Vida de los Mayores (Instituto de Mayores y Servicios Sociales. 2004. Estudio 4597). En el estudio europeo ENABLE-AGE la tasa de no respuesta en el caso alemán es del 22,6\% (Oswald et al. 2005). 


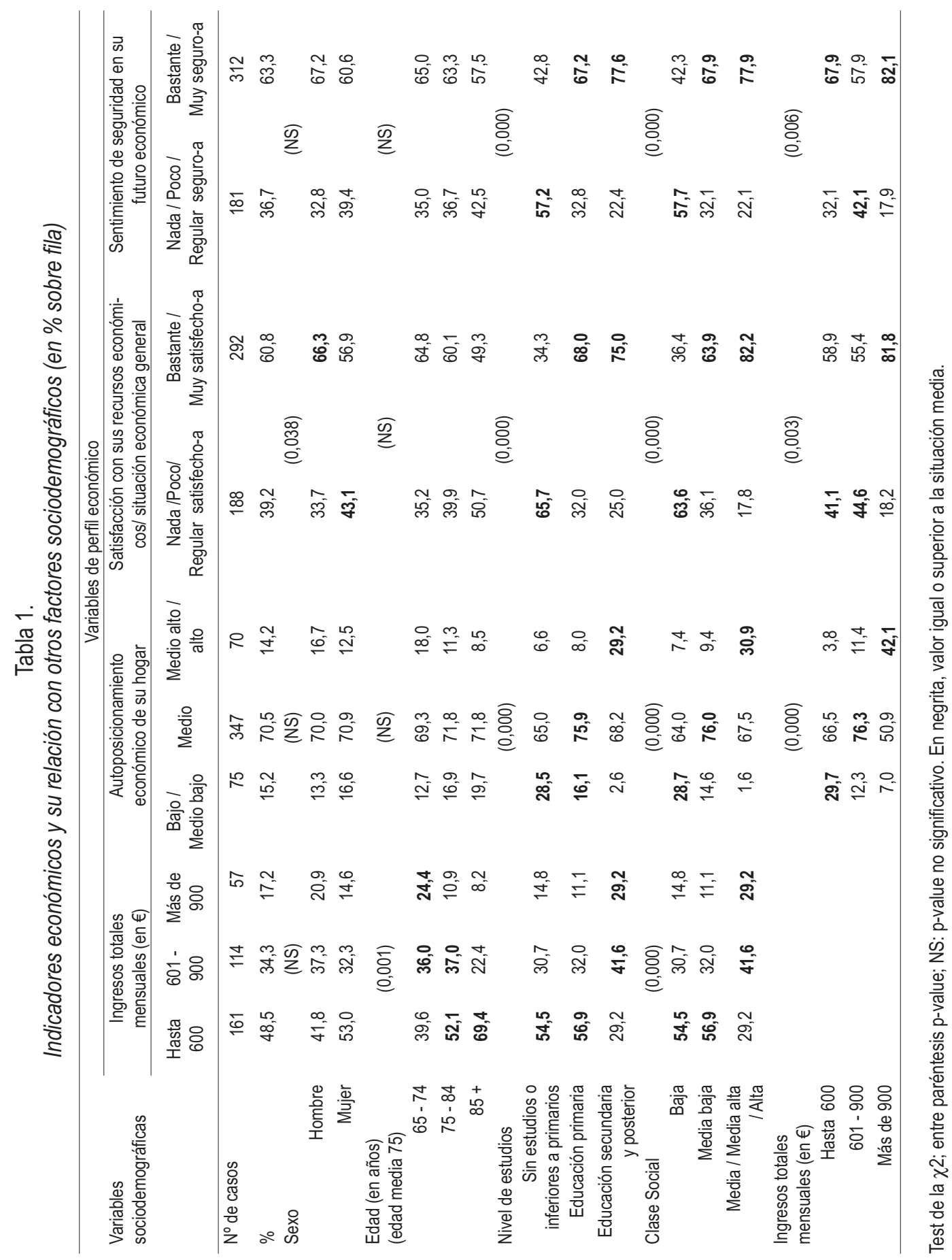


La edad, el nivel de estudios y la clase social son las variables que mejor definieron la distribución de los ingresos económicos, ya que entre los que declaran menor renta mensual $(<600 €)$ tienden a predominar quienes tienen más edad, estudios primarios o inferiores y, consecuentemente, una clase social por debajo de la media; mientras que los que perciben rentas más elevadas suelen alcanzar un mayor nivel de estudios (secundarios y superiores) y una clase social media o superior. Esta relación significativa se mantiene cuando se pregunta por el autoposicionamiento socioeconómico del hogar detentador de ingresos (tabla 1), de forma que a medida que aumentan los ingresos individuales obtenidos se incrementa la posición económica percibida del hogar, también más elevada entre quienes tienen mayor nivel de estudios y clase social.

Los ingresos provienen casi exclusivamente de una sola fuente (en el 96\% de los casos), pensiones de jubilación o de viudedad, las más comunes entre la población mayor, esencialmente entre quienes menor nivel de ingresos declaran, tendencia tradicional entre los mayores españoles (Pérez Ortiz 1997). Así, más de 9 de cada 10 hombres, por 5 de cada 10 mujeres, reciben pensión de jubilación; mientras un $45 \%$ de las mujeres que han respondido a la pregunta sobre ingresos reciben una pensión de viudedad, en 9 de cada 10 casos como única fuente, casi en su totalidad viudas, por casi ningún hombre. El tener una edad superior a los 75 años, pertenecer a clases sociales media o baja, y haber conseguido un nivel de estudios primarios son elementos del perfil sociodemográfico de los perceptores de las pensiones básicas. El escaso número de encuestados que refieren más de una fuente de ingresos (sólo un 4\%) muestra una combinación entre dos tipos de pensiones, resultado de su historia laboral. Apenas un 1,5\% de la población madrileña recibe una pensión de jubilación y declara tener, además, otros ingresos patrimoniales, como consecuencia de una acumulación de medios económicos durante su vida laboral.

La medida de conceptos tan difíciles de aprehender como la "satisfacción con" o la "seguridad en" la situación económica no es fácil. Algunos estudios han utilizado solamente una pregunta para ahondar en la satisfacción o en la seguridad, confiando en la capacidad de los entrevistados para comprender el significado preciso de tales conceptos (Pérez Ortiz 2006). En otros casos se investigan "satisfacción" y "seguridad" a través de constructos formados por otras realidades como la capacidad para hacer frente a emergencias o necesidades, la compra de determinados bienes para obtener una "satisfacción económica" (Lamura 2003), o la capacidad de ahorro cada mes (Instituto de Mayores y Servicios Sociales 2004). También puede usarse la "seguridad material" o la "adecuación material" (Hawkins 2005), como indicadores basados en la disposición de recursos suficientes para asegurar el mantenimiento físico, la disponibilidad de vivienda y el acceso a los servicios, esenciales para la promoción de un adecuado estado de salud en la vejez. 0 , simplemente, el sentirse bien y libre para afrontar posibles emergencias con los recursos disponibles (Gabriel y Bowling 2004), cuando la equiparación a la baja de los ingresos de los mayores, obtenidos de las pensiones, hace que su percepción sea superior a la valoración real de los mismos (Bowling y Gabriel 2004). Se llega entonces a plantear la "paradoja de los recursos económicos" entre los mayo- 
res (Weidekamp-Maicher y Naegele 2007) al subestimar la importancia de los recursos económicos objetivos condicionada por una satisfacción subjetiva, a su vez, influida por otros factores. El riesgo de caer en la exclusión social y en la pobreza es evidente para determinadas capas de la población mayor si existe una falta de adecuación entre recursos y necesidades. Pero, desde un punto de vista perceptual, esta adecuación introduce otro elemento a ser tenido en cuenta: la adaptación a la situación económica en la que se vive como mecanismo de supervivencia, muy común entre los mayores; lo que les permite "ajustarse", tal y como lo han estado haciendo a lo largo de su vida, a bienes primarios (Bazo 1990). Otras necesidades secundarias (equipamientos de la vivienda, actividades de ocio y cultura) si se ven comprometidas en las decisiones de gasto de los mayores españoles (Pérez Ortiz 2006). La percepción de algunas de estas necesidades no cubiertas tiende a reforzar las opiniones negativas con respecto a la CdV individual y a la percepción de la salud, como su referente fundamental.

Bajo el contexto previo, la visión subjetiva global de los recursos disponibles se ha indagado a través de dos cuestiones sencillas, sobre la "satisfacción con los recursos económicos" y la "seguridad del futuro económico", al margen de su relación con las necesidades que se precisan cubrir a esta edad, como tendencia seguida en los proyectos European Study on Ageing Well-ESAW (Lamura et al. 2003) y Growing Older (Bowling et al. 2002).

En general, en torno a 6 de cada 10 mayores declaran estar bastante o muy satisfechos con sus recursos económicos, y en la misma proporción se sienten bastante 0 muy seguros de su futuro económico (tabla 1), siendo los hombres los que muestran mayor satisfacción. No se encontró, en cambio, asociación estadística entre estas evaluaciones y la edad, aunque la tendencia muestra más satisfacción o seguridad entre los viejos-jóvenes (menores de 75 años). Por su lado, el nivel de estudios más alto conseguido y la clase social se asocian con estas variables subjetivas, de modo que los que alcanzan mayores niveles también están más satisfechos con sus recursos o seguros de su futuro económico.

Se ha observado también una asociación estadística significativa en la relación entre el nivel de ingresos y su evaluación en términos de satisfacción y seguridad, de manera que a mayor nivel de ingresos mejores valoraciones. Sin embargo, entre los que declaran menores ingresos, en general receptores de pensiones no contributivas, existe una proporción destacable de personas con un cierto sentimiento de conformidad, de adecuación de las necesidades a los ingresos disponibles, así como, previsiblemente, de superación de unas expectativas negativas previas al recibir unos ingresos que no esperaban. Este comportamiento ha sido definido, según la terminología de Zapf (Noll 2002), como de "adaptación" a unas malas condiciones objetivas de vida, en este caso determinadas por un bajo nivel de ingresos económicos, expresada mediante un relativamente alto nivel de satisfacción o bienestar subjetivo. En el otro extremo, en torno a un $18 \%$ de los mayores declara ingresos mensuales elevados pero manifiestan un cierto nivel de insatisfacción, en una clara "disonancia" (Noll 2002), quizás ligada con unas necesidades superiores a las habitualmente requeridas a esta edad y heredadas de la 
etapa adulta, y unas expectativas no siempre cumplidas. Esa insatisfacción es incluso más apreciable cuando los ingresos son algo inferiores (entre 601 euros y 900 euros) (tabla 1).

El nivel de estudios y la clase social, como expresión máxima de la trayectoria económica personal a lo largo de la vida, se encuentran, de nuevo, entre los aspectos condicionantes del comportamiento perceptual. Partiendo de la base de que el nivel de estudios es un factor "capacitador" de la consecución de ingresos económicos durante la etapa laboral y, consecuentemente, de la pensión durante la jubilación, es razonable suponer que la valoración de los recursos económicos sea percibida de forma desigual según los diferentes grados formativos. Así, los niveles más bajos de satisfacción con la situación económica general en el momento presente y de seguridad en el futuro económico (tabla 1) son expresados por quienes menores niveles formativos y de clase social declaran; de modo que en torno a dos tercios de aquellos que no han superado los estudios primarios o tienen clase social baja declaran un nivel de satisfacción y seguridad asimismo bajos (nada, poco o regular); mientras que también alrededor de dos tercios de quienes tienen educación primaria, secundaria o superior o clase social media o superior han manifestado un nivel alto de satisfacción con sus recursos y de seguridad en su futuro económico. No obstante, en este punto hay que recordar que entre los mayores madrileños es escasa la proporción de personas con estudios superiores $(2,5 \%)$ y de clase social alta $(1,2 \%)$, lo que reduce las posibilidades de extraer opiniones fundadas de tales números.

\section{UNA TIPOLOGÍA DE LOS MAYORES SEGÚN SUS RECURSOS ECONÓMICOS}

La relación entre los datos objetivos y las opiniones subjetivas de los encuestados sobre su situación económica se ha establecido por medio de la técnica estadística del Análisis Factorial, por el método de componentes principales (tabla 2). Dada la previsible relación entre ambas magnitudes, el resultado es expresivo de la formación de dos factores que, en su conjunto, explican el $80,6 \%$ de toda la variabilidad, siendo la satisfacción con sus recursos económicos y la seguridad ante su futuro económico las variables que conforman el primer factor (percepción), mientras que sus ingresos totales y el autoposicionamiento del hogar forman el segundo factor (ingresos).

Tan importante como comprobar la interrelación de las variables de tipo económico en su doble perspectiva objetiva-subjetiva es la clasificación de sujetos de acuerdo con los dos componentes principales latentes obtenidos. Tomando en cuenta las respuestas efectivas a las preguntas sobre ingresos, se han identificado 4 grupos de personas mayores definidos por su posición en relación con dichos componentes principales. En primer lugar, casi un $29 \%$ de los mayores (Figura 1) han sido clasificados como "optimistas con base económica", al confluir buenas condiciones económicas objetivas (ingresos mensuales y autoposicionamiento del hogar) y evaluaciones subjetivas positivas, en una situación que, según la tipología de CdV basada en la combinación de condiciones obje- 
Tabla 2.

Relación subyacente entre las variables objetivas y subjetivas sobre la situación económica a partir de Análisis Factorial.

\begin{tabular}{lccc}
\hline & \multicolumn{2}{c}{ Componentes y pesos factoriales (a) } \\
\cline { 2 - 3 } & Comunalidades & $\begin{array}{c}\text { Seguridad en su } \\
\text { futuro económico y } \\
\text { satisfacción con sus } \\
\text { recursos económicos } \\
\text { (percepción) }\end{array}$ & $\begin{array}{c}\text { Ingresos totales } \\
\text { mensuales y } \\
\text { autoposiciona- } \\
\text { miento económico } \\
\text { del hogar } \\
\text { (ingresos) }\end{array}$ \\
$\begin{array}{l}\text { Variables } \\
\begin{array}{l}\text { Seguridad en su futuro } \\
\text { económico }\end{array}\end{array}$ & 0,845 & $\mathbf{0 , 9 1 8}$ & \\
$\begin{array}{l}\text { Satisfacción con sus recursos } \\
\text { económicos, su situación } \\
\text { económica general }\end{array}$ & 0,833 & 0,904 & $\mathbf{0 , 9 1 8}$ \\
$\begin{array}{l}\text { Ingresos totales mensuales } \\
\begin{array}{l}\text { Autoposicionamiento económico } \\
\text { del hogar }\end{array}\end{array}$ & 0,848 & 0,461 & $\mathbf{0 , 6 9 5}$ \\
\hline
\end{tabular}

(a) Se muestran las puntuaciones factoriales $\geq 0,300$ en valor absoluto.

Método de Extracción: Análisis de Componentes Principales.

Método de Rotación: Varimax con Normalización Kaiser (la rotación ha convergido en 3 iteracciones).

Varianza total explicada: 80,55

Medida de adecuación muestral Kaiser-Meyer-Olkin: 0,593

Test de esfericidad de Bartlett: Chi-Square 632,001 Sig.: 0,000; gl: 6

Determinante de la matriz de correlaciones: 0,326

tivas de vida y bienestar subjetivo se define como "bienestar" (Noll 2002). Un segundo grupo ha sido denominado aquí como "optimistas sin base económica" (cerca del 30\%), cuya visión positiva no se fundamenta en los recursos económicos que afirman tener sino, quizás, en otros factores de tipo social, familiar o asociados con el entorno en el que viven. A este respecto diversos autores sugieren que valoraciones positivas frente a condiciones de precariedad podrían explicarse por el hecho de que con la edad se llegan a aceptar estas situaciones por un cambio de valores (Cohn y Sugar 1991; Lawton 1991) en un proceso de "adaptación" a circunstancias negativas de vida (Noll 2002). El tercer grupo, casi un $22 \%$ de los individuos, son clasificados como "pesimistas sin base económica", cuyo perfil se define por un nivel de ingresos inferior a la media y por una percepción negativa de sus recursos y seguridad en su futuro económico, en una posición de "privación" (Noll 2002). Finalmente, el cuarto grupo es el denominado aquí como "pesimistas con base económica", casi otro $20 \%$ de los individuos que, al igual que el 
grupo anterior, no tienen una percepción positiva de su situación económica, pero que declaran recursos superiores a la media, y suficientes como para que su visión no fuera negativa o de "disonancia" (Noll 2002).

Al no haberse incluido una pregunta específica en la encuesta, no es posible relacionar las necesidades de las personas mayores con sus ingresos económicos pero, de una forma indirecta y aproximada, se pueden conocer estas "necesidades" a través de la indagación de los ámbitos de la vida de las personas en los que la sociedad, en su conjunto, debería hacer más inversiones (tabla 3). Las máximas preocupaciones de los mayores se centran en conseguir que la sociedad mantenga las "pensiones", un $75 \%$ de los encuestados cita este aspecto, especialmente aquéllos clasificados como optimistas sin base económica, es decir, los que tienen un menor nivel de ingresos pero una percepción positiva de los mismos, en el interés de seguir manteniendo esa confianza en sus recursos económicos. En cambio, una proporción inferior de los que reciben más ingresos eligieron esta respuesta (69\%). También se menciona de forma mayoritaria la necesidad de invertir en más recursos sanitarios (73\%), en este caso esencialmente los que mejor percepción tienen de su situación, aunque no reciban tantos ingresos (optimistas sin base económica: $76 \%$ ), en contraposición a quienes se encuentran en una situación de confluencia entre bajos ingresos y percepción negativa de su situación

Figura 1.

Clasificación de sujetos según los recursos económicos y su valoración.

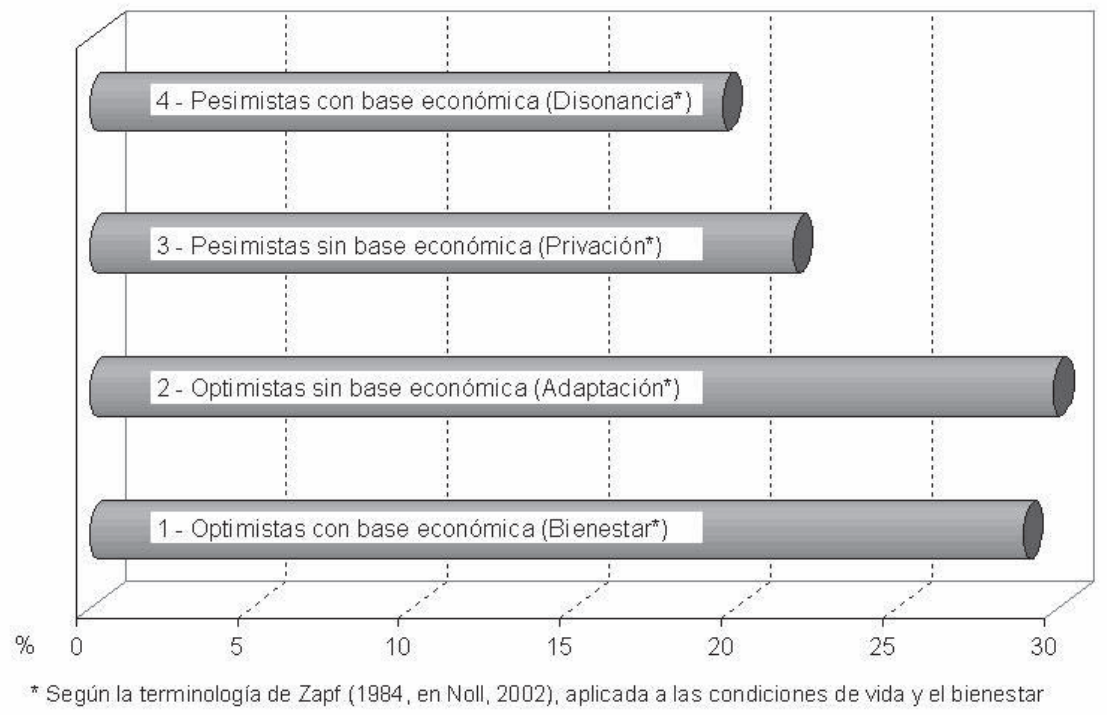


económica (pesimistas sin base económica: 57\%). Pensiones e inversiones en sanidad son ámbitos de referencia esenciales para los individuos, ya que los ingresos procedentes de las pensiones se destinan a asegurar la mejor cobertura de las necesidades individuales, sobre todo para aquellas personas que menos valor aprecian en ellos, a la vez que se demandan recursos "en especie" para atender los problemas de salud como principal inquietud de los mayores en su proceso de envejecer. A una distancia muy apreciable son mencionadas también las inversiones en "vivienda" (37\%), otro hecho de amplio interés social, especialmente declarado por los mayores que menores recursos tienen y que, en términos proporcionales, suelen vivir en régimen de alquiler, y la "educación" (27\%), quizás una reclamación sentida por muchos mayores que no tuvieron la oportunidad de conseguir una formación adecuada para una mejora de su situación económica.

\section{LOS FACTORES EXPLICATIVOS DE LA DIMENSIÓN "RECURSOS ECONÓMICOS"}

Las condiciones económicas de las personas mayores, medidas objetiva y subjetivamente, están relacionadas con un conjunto de variables que contribuyen a definir su estilo de vida, su forma de vivir en familia y de envejecer en su entorno residencial, en su sistema de relaciones sociales, en su comportamiento diario. Como elementos que conforman su estructura vital en el presente, se han ido construyendo a lo largo de varias décadas, de manera que se valoran cuando se disfrutan como la herencia recibida de su etapa adulta, la formación de la familia, la vida laboral y la construcción de su entorno físico y social. Se ha pretendido, pues, avanzar en la evaluación de la situación económica de los mayores de acuerdo con aquellos aspectos que cumplen la condición de tener una relación estadística significativa con la tipología de individuos según sus recursos económicos, tal y como ha sido establecida en el epígrafe anterior.

Entre estos aspectos, la formación educativa recibida antes de la entrada en la vida laboral (asociada a lo largo de ésta con la condición social conseguida) es la principal variable sociodemográfica relacionada con la tipología de mayores definida según las condiciones económicas objetivas y subjetivas. Su grado de significación es alto $\left(\chi^{2}\right.$ : $65,9 ; p$-value $\leq 0,0001)$, ya que cerca de un $50 \%$ de quienes tienen mayor nivel de estudios (secundarios y superiores) son los que declaran más altos ingresos y son los que mejor visión de la vida tienen, esto es, los "optimistas con base económica". Por el contrario, entre los que no saben leer ni escribir o no terminaron sus estudios primarios, un $42 \%$, son "pesimistas sin base económica", abocados a situaciones previsibles de "privación", especialmente si las condiciones económicas, individuales y familiares se extreman. En una situación de transición entre las anteriores se encuentran los que declaran estudios primarios, quienes en un $41 \%$ de los casos son clasificados como "optimistas sin base económica". 
Tabla 3.

Ámbitos de la vida en los que la sociedad debería invertir más dinero.

\begin{tabular}{lccc}
\hline Invertir en & $N^{0}$ respuestas & \% respuestas & $\begin{array}{c}\text { \% casos } \\
\text { (493 casos válidos) (1) }\end{array}$ \\
\hline Pensiones & 372 & 25,3 & 75,5 \\
Sanidad & 361 & 24,5 & 73,2 \\
Vivienda & 180 & 12,2 & 36,5 \\
Educación & 134 & 9,1 & 27,2 \\
Empleo & 110 & 7,5 & 22,3 \\
Seguridad ciudadana & 66 & 4,5 & 13,4 \\
Infraestructuras: carreteras, calles & 60 & 4,1 & 12,2 \\
Inmigrantes & 55 & 3,7 & 11,2 \\
Justicia & 42 & 2,9 & 8,5 \\
Familia & 36 & 2,4 & 7,3 \\
Paz y seguridad en el mundo & 32 & 2,2 & 6,5 \\
Lucha contra la desigualdad social & 17 & 1,2 & 3,4 \\
Medioambiente & 6 & 0,4 & 1,2 \\
Total & 1.471 & 100,0 & 298,4 \\
\hline
\end{tabular}

(1) Al solicitarse tres respuestas por orden de importancia para el encuestado, esta pregunta se ha tratado como 'multirrespuesta', con resultados numéricos más altos que el número de personas encuestadas puesto que cada una de ellas puede dar varias respuestas.

En relación con la edad, las categorías especificadas no presentan una relación muy clara, con una significación estadística moderada $\left(\chi^{2:}: 12,7 ; p\right.$-value $\left.\leq 0,05\right)$, pero se identifican dos asociaciones interesantes: cerca de un $60 \%$ de los viejos-jóvenes (con menos de 75 años) son esencialmente "optimistas", independientemente de los recursos que reciben, mientras que casi un $70 \%$ de los mayores de 85 años carecen de base 0 recursos económicos suficientes, independientemente de su percepción. Los años que separan a ambos grupos etarios son, probablemente, reflejo de la "adaptación" a unas condiciones de vida actual en situaciones muy distintas.

Otras variables sociodemográficas como el sexo o el tipo de hogar no muestran relaciones estadísticas significativas con la tipología de mayores definida por sus recursos económicos, a partir de la información obtenida en este trabajo. Como se mencionó anteriormente, hay evidencias científicas que sugieren que la influencia del género, como constructo social, queda enmascarada en otras variables sociodemográficas como factores predictores del comportamiento económico de la población, a pesar de que haya diferencias en la posición económica de hombres y mujeres. 
Por lo que respecta al estado de salud, las investigaciones internacionales sobre CdV atestiguan de forma clara su asociación con los recursos económicos disponibles (Grundy y Sloggett 2003; Andresen y Millar 2005; Higgs et al. 2005; Evans et al. 2008), con componentes ligados a la dimensión temporal (Smith y Kington 1997; Meer et al. 2003; Berry 2007) y con aspectos psicosociales de la vida del individuo (Taylor y Seeman 1999) como atributos muy destacados. En general, se cumple la conocida ley que señala que a mayor nivel económico mejor evaluación subjetiva del estado de salud, incluso marcándose algunas diferencias en espacios geográficos con similares situaciones demográficas y sociopolíticas (Tsimbos 2010), y ambas dimensiones condicionando de forma positiva la CdV referida (tabla 4), de la misma manera que se comprueba con otros aspectos relacionados con la salud, como la discapacidad (Alfageme 2001).

Tabla 4.

Proporción de personas mayores según sus recursos económicos y estado de salud (en \% sobre fila).

\begin{tabular}{lcccc}
\hline & $\begin{array}{c}\text { Optimistas } \\
\text { con base } \\
\text { económica } \\
\text { (Bienestar) }\end{array}$ & $\begin{array}{c}\text { Optimistas } \\
\text { sin base } \\
\text { económica } \\
\text { (Adaptación) }\end{array}$ & $\begin{array}{c}\text { Pesimistas } \\
\text { sin base } \\
\text { económica } \\
\text { (Privación) }\end{array}$ & $\begin{array}{c}\text { Pesimistas } \\
\text { con base } \\
\text { económica } \\
\text { (Disonancia) }\end{array}$ \\
\hline Muy malo / malo ( $\mathrm{n}=19)$ & 15,8 & 0,0 & $\mathbf{5 2 , 6}$ & $\mathbf{3 1 , 6}$ \\
Regular ( $\mathrm{n}=77)$ & 22,1 & 20,8 & $\mathbf{3 3 , 8}$ & $\mathbf{2 3 , 4}$ \\
Bueno $(\mathrm{n}=177)$ & 35,6 & 29,4 & 15,8 & 19,2 \\
Muy bueno ( $\mathrm{n}=48)$ & 20,8 & $\mathbf{5 8 , 3}$ & 12,5 & 8,3 \\
Total $(\mathrm{n}=321)$ & 29,0 & 29,9 & 21,8 & 19,3 \\
\hline
\end{tabular}

Test de la $\chi 2: 50,3 ; \quad$ gl: 9; $\quad$-value $\leq 0,001$

En el caso de los mayores de la Comunidad de Madrid, esa relación es estadísticamente significativa y se establece entre quienes, independientemente de la base económica que tengan, no hacen una valoración positiva de su economía, los más "pesimistas", y los que perciben un estado de salud que tiende a ser considerado de regular a muy malo. Son apenas un $12 \%$ del total de la población mayor madrileña, pero más del $80 \%$ de los que manifiestan un peor estado de salud. Por el contrario, una actitud positiva hacia los recursos económicos ("optimistas") está asociada con valoraciones también positivas del estado de salud, ya que, de entre los que contestan que gozan de buena o muy buena salud, casi dos tercios en el primer caso y cerca del $80 \%$ en el segundo manifiestan 
una visión optimista de sus recursos. Es sintomático que el 56,4\% de los que declaran tener mejor salud son "optimistas con base económica", la situación ideal para disfrutar del bienestar económico en la vejez en las mejores condiciones de salud.

La dimensión que explora las redes de apoyo de los mayores tiene, también, una apreciable representación en estudios sobre $\mathrm{CdV}$, relaciones sociales/redes de apoyo y salud (Achat et al. 1998; Michael et al. 1999; Pinquart y Sorensen 2000; Jang et al. 2004; Rojo-Pérez et al. 2009a; 2009b). En la encuesta CadeViMa esta relación ha sido analizada por medio de dos variables: una es la "necesidad o no de ayuda" de personas cercanas (vivan o no en el domicilio del mayor) para realizar las actividades de la vida diaria (AVD); otra es la "persona de quien el mayor recibe la ayuda". En buena lógica esta necesidad debe estar relacionada con el nivel de competencia relacionado con la salud y el funcionamiento. Mientras en la primera variable (necesidad de ayuda) se establece una asociación significativa desde el punto de vista estadístico $\left(\chi^{2}: 23,3 ; p\right.$-value $\leq$ $0,001)$ con los recursos económicos, en el segundo caso (persona que ayuda) no es así.

La primera constatación es que los tipos de mayores definidos según sus recursos económicos se diferencian significativamente ( $p$-value $\leq 0,0001)$ de acuerdo con la recepción de ayuda para el desempeño de AVD (Figura 2). Se identifica así una proporción del $80 \%$ de mayores que no tienen esa necesidad (nodo 1), de los que aproximadamente dos tercios valoran positivamente su situación económica: son los más "optimistas", ya sea por tener recursos económicos suficientes, ya sea por su "capacidad de adaptación" a su situación actual y, previsiblemente, a la futura. Por el contrario, de entre quienes tienen necesidad de ayuda (nodo 2, el 20\% restante) alrededor del $47 \%$ son "pesimistas sin base económica", lo que les sitúa en una posición de "privación". En ambos casos, necesitar o no ayuda, se muestra una asociación estadística significativa con el estado de salud percibido ( $p$-value $\leq 0,01)$. Así, de entre aquéllos que no necesitan ayuda y declaran un estado de salud bueno (nodo 4), más del $70 \%$ son mayores "optimistas", con base económica o no, mientras que éstos suponen sólo un $42 \%$ de los que perciben un estado de salud malo (nodo 3). Por otro lado, de entre quienes necesitan ayuda y declaran un mal estado de salud (nodo 5), en torno al $72 \%$ son mayores con una valoración negativa ("pesimistas" con o sin base económica) de sus condiciones económicas, porcentaje que se reduce al $50 \%$ cuando se percibe un estado de salud bueno (nodo 6). Por lo tanto, el estado de salud, como condición de predisposición y necesidad de ayuda, como exigencia a cubrir, tiene una asociación muy definida con los recursos económicos.

Otros factores relacionados con los recursos económicos son la existencia o no de una red social formada por amigos, más allá de las personas conocidas en el entorno en el que se vive (Baxter et al. 1998), la frecuencia de relación con estos amigos, y el grado de satisfacción con la forma de vivir en su actual situación familiar; todos estos factores muestran asociación estadística significativa ( $p$-value $\leq 0,0001)$ con el perfil económico de los mayores. Dentro de este contexto, la satisfacción con la forma de vivir es la variable dominante en esa relación (Figura 3), con una elevada significación estadística ( $p$-value $\leq 0,0001$ ), destacando los encuestados satisfechos (nodo 1, un $81 \%$ del total 


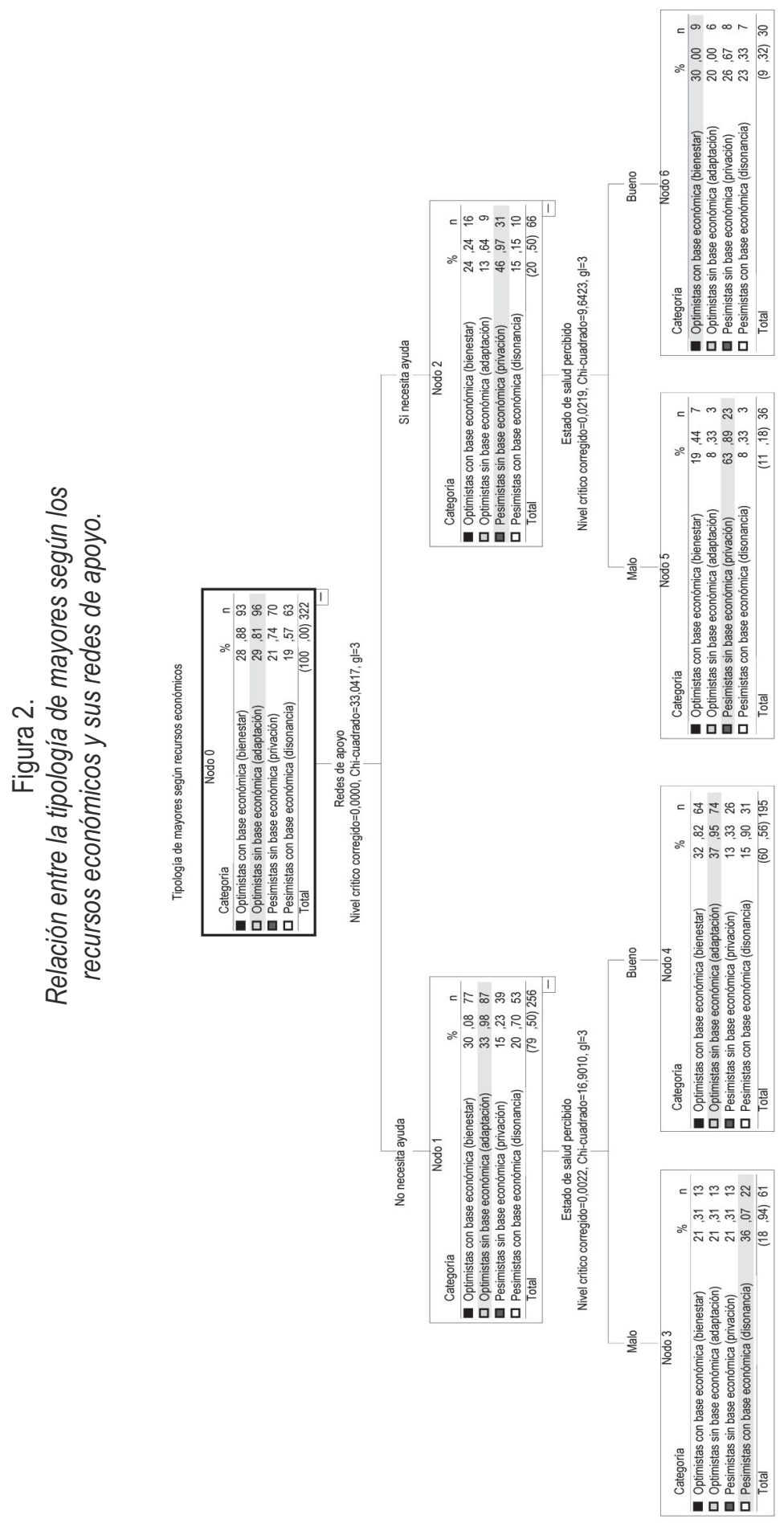


de mayores) que son los que mejor valoran sus recursos económicos (los "optimistas" representan aproximadamente dos tercios), frente a los insatisfechos (nodo 2, un 19\% del total) con un $72 \%$ de "pesimistas". Para los satisfechos con la forma de vivir, la siguiente variable significativa es la frecuencia de relaciones con la red social ( $p$-value $\leq$ 0,01 ), distinguiendo entre quienes se relacionan 1 ó 2 veces por semana o más (nodo 3 , un $66 \%$ del total de mayores) y 1 ó 2 veces al mes o menos (nodo 4 , un $15 \%$ del total). El $70 \%$ de los primeros se declara "optimista" respecto a su situación económica, en un comportamiento muy distinto a quienes mantienen una baja frecuencia de relaciones, de los que un $54 \%$ son "pesimistas" en lo económico. La última variable significativa de este modelo es el tamaño del hogar (nodos 5,6 y 7 , con el $10 \%, 33 \%$ y $24 \%$ del total de personas mayores, respectivamente), de modo que entre quienes se declaran satisfechos con su forma de vivir (nodo 1) y mantienen una relativamente elevada frecuencia de relaciones de amistad (nodo 3), el hecho de vivir en hogares de 2 personas (nodo 6) introduce un contexto de refuerzo para el buen desarrollo de las condiciones de vida y el mejor uso de los recursos económicos en relación con las necesidades detectadas (Pérez Ortíz 2006). Así, casi el 70\% de los que viven en hogares de 2 miembros (nodo 6), es decir, el mayor con otra persona, previsiblemente su pareja, es "optimista", pero también lo son 8 de cada 10 mayores que viven solos (nodo 7), frente a los 5 de cada 10 que viven en hogares de 3 o más personas (nodo 5). En definitiva, los "optimistas", con 0 sin base económica, tienden a ser, además, individuos satisfechos con su forma de vivir y con la frecuencia de relaciones con su red social.

Ocio y tiempo libre es otra dimensión de la CdV (Fernández-Mayoralas et al. 2007; Lardiés-Bosque et al. 2007) a explorar en relación con los recursos económicos, porque permite apreciar tanto la capacidad económica directa para la realización de algunas actividades (viajes, prácticas culturales, uso de nuevas tecnologías,...) como la predisposición personal a llevar a cabo otras que implican retos personales, aunque ello no siempre tenga que ver con la economía personal o familiar, sino con otros factores sociales y de salud (Warr et al. 2004; Li 2005). No es fácil llegar a un acuerdo sobre los mejores instrumentos de análisis de las actividades de tiempo libre, porque hay un amplio elenco de medidas que podrían ser utilizadas (Brown et al. 2004), pero una discriminación entre actividades "pasivas" y "activas" es necesaria para separar aquellas tareas con escasos requerimientos personales de otras más demandantes de recursos materiales, de movilidad o de simple predisposición, pero que aportan mayor adecuación a la CdV declarada (Milligan et al. 2004). El análisis previo de la información sobre actividades de ocio (Lardiés-Bosque et al. 2007) permitió destacar sólo tres tipos de actividades por su relación con los recursos económicos y su valoración: son las de "participación en ocio activo y social" (salir con amigos/as a pasear, asistir a eventos deportivos, participar en actividades asociativas, realizar actividades organizadas en la parroquia), los "viajes y turismo", y las de "carácter pasivo" (ver TV, escuchar la radio, escuchar música, ver vídeos).

En relación con esta dimensión, la primera variable estadísticamente significativa 


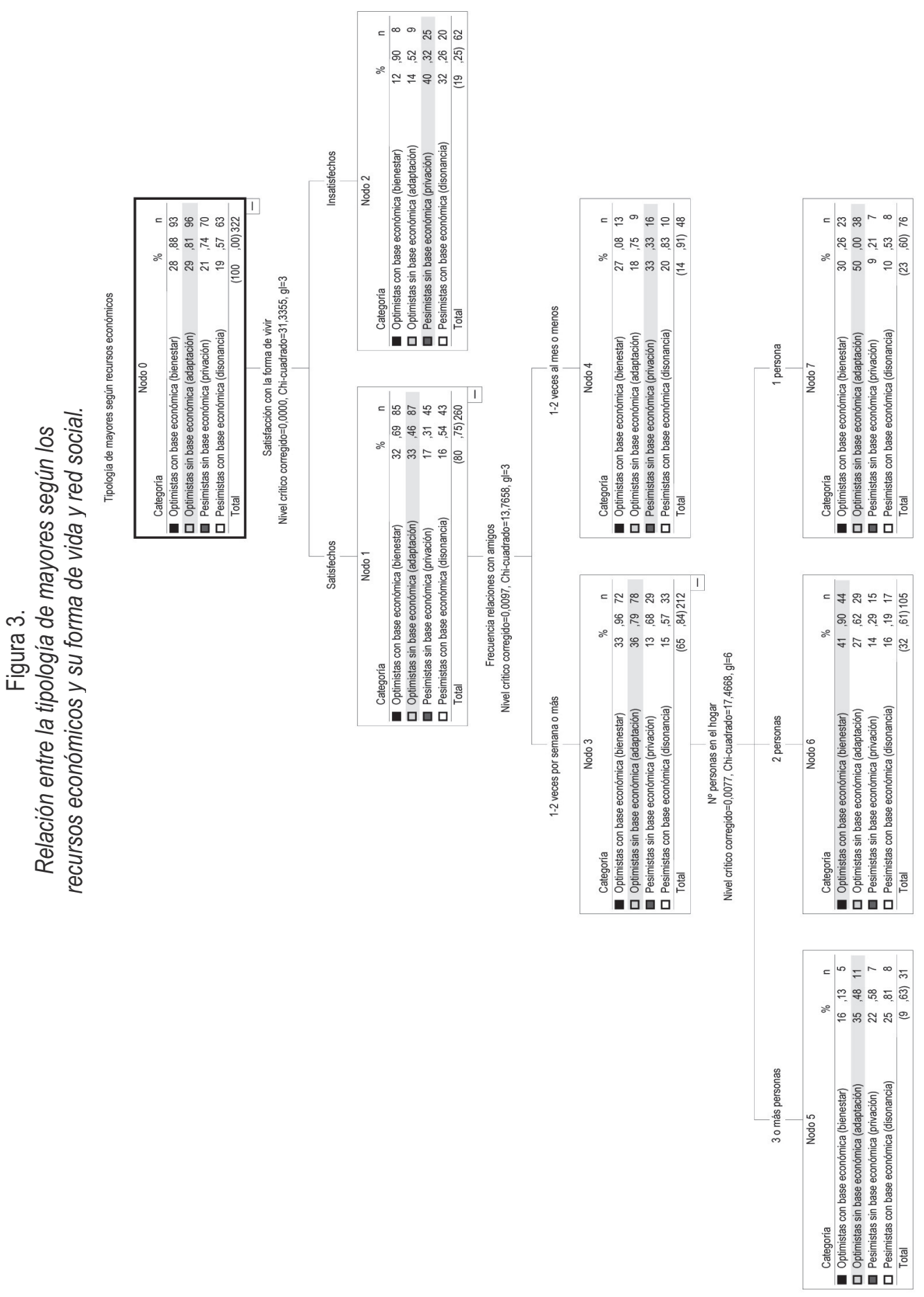



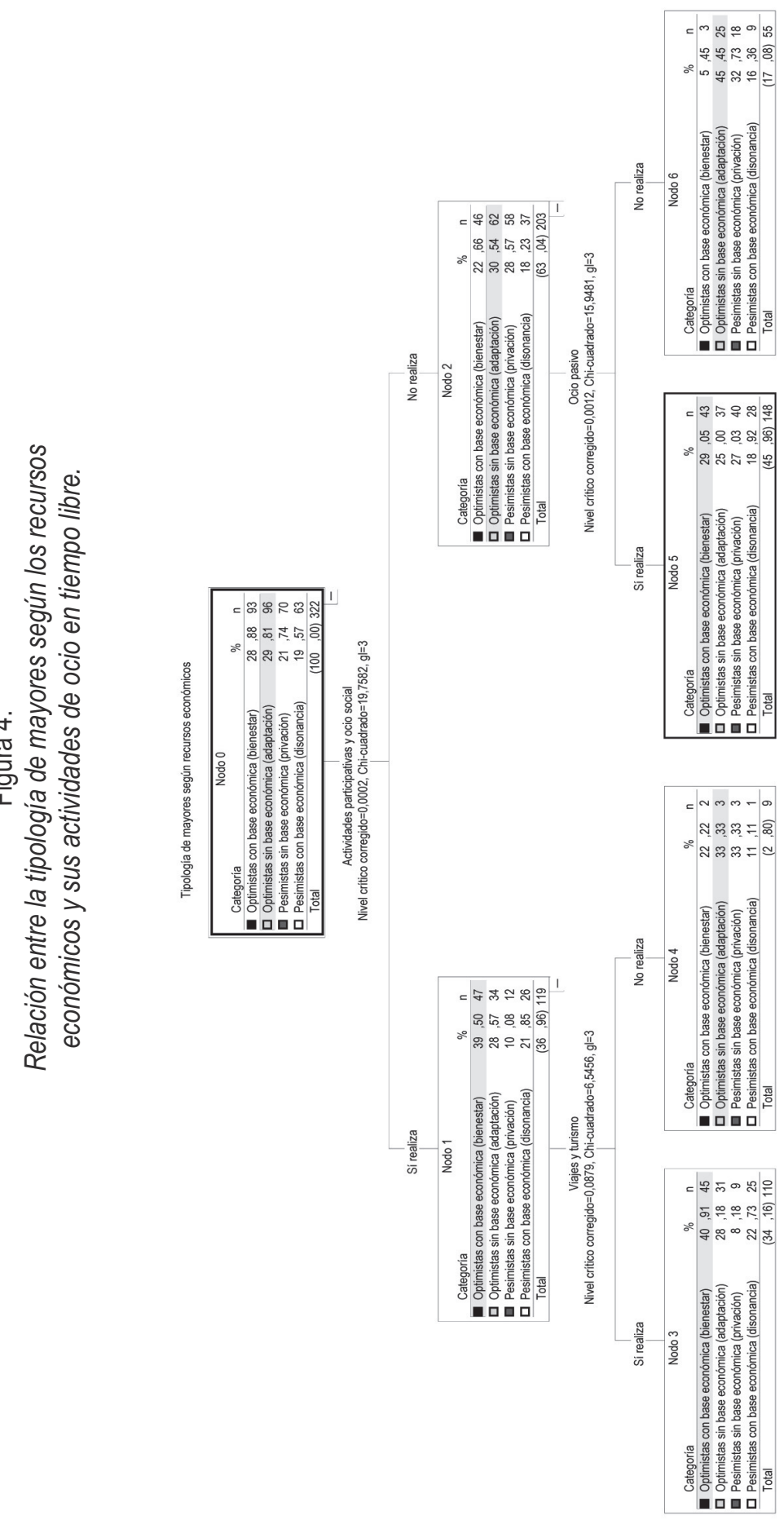

RIS, VOL.69. No 1, ENERO-ABRIL, 193-225, 2011. ISSN: 0034-9712. DOI: 10.3989/ris.2009.11.26 
con la tipología de mayores según los recursos económicos es la realización de actividades denominadas como participativas y ocio social ( $p$-value $\leq 0,001)$ (Figura 4). Así, un $37 \%$ del total de mayores declaran practicar estas actividades (nodo 1) y muestran, no sólo una tendencia a tener "base económica", bien sea ésta valorada de forma positiva o negativa $(60 \%)$, sino también una visión "optimista" hacia sus recursos económicos disponibles (68\%). Los mayores involucrados en ocio de carácter participativo y social también lo están en actividades relacionadas con el turismo, las excursiones y los viajes (nodo 3), si bien esta asociación tiene una significación estadística muy débil $(p$-value $=0,09)$.

Por su lado, más de 6 de cada 10 personas no realizan ocio participativo y social (nodo 2), tratándose, principalmente, de mayores "sin base económica", bien optimistas, bien pesimistas. Este perfil de mayores se asocia estadísticamente ( $p$-value $\leq 0,01)$ con las actividades de ocio denominadas como pasivas (nodos 5 y 6 ), siendo así que el $73 \%$ de los mismos declara estar involucrado en estas actividades como tipología de ocio muy generalizada entre los mayores. La realización de ocio de tipo pasivo es expresiva de una forma de vida en la vejez contraria a una participación en ocio activo (actividades sociales, viajes, turismo, deportes) lo que, en cierta medida, requiere un mayor consumo de recursos económicos.

Finalmente, hay un factor esencial más en la vida de las personas mayores, el entorno geográfico y social donde se desarrolla la vida, principalmente la casa y el barrio de residencia, que actúa como elemento expresivo de una situación económica más o menos favorable (Rojo-Pérez et al. 2000; Bowling y Gabriel 2004; Fernández-Mayoralas et al. 2004). Se trata de una condición material (el capital económico, la vivienda) y/o inmaterial (la satisfacción por vivir en el barrio), que se consigue acumular durante el ciclo de vida y que cada persona interpreta según sus rasgos personales y su disposición cognitiva, y la introduce como referencia en su comportamiento diario. De entre las variables que pueden ser utilizadas en este ámbito, se han destacado tres por su relación significativa con los recursos económicos de los mayores: la satisfacción con el barrio, el índice de calidad global de la vivienda (como indicador del nivel y tipo de dotaciones o equipamiento en la casa y en la finca, incluyendo régimen de tenencia, antigüedad y superficie) (Rojo-Pérez et al. 2007) y el número de reformas de mantenimiento necesarias en la vivienda, que marca la situación de necesidad de afrontar las condiciones de habitabilidad en relación con los recursos disponibles.

En este contexto, un hecho destacado en la evaluación de los recursos residenciales con los que cuentan los mayores es que casi un $90 \%$ de ellos se encuentran satisfechos de vivir en su barrio o pueblo (Figura 5). Y, como era de esperar, se trata de una relación altamente significativa ( $p$-value $\leq 0,0001)$ porque se basa en una clara valoración de las personas que aprecian positivamente su posición económica personal (los "optimistas" en el nodo 1) como aspecto relacionado con su satisfacción por vivir en ese entorno; de la misma manera que entre los no satisfechos (nodo 2) la relación se establece con los "pesimistas", en ambos casos sin que los ingresos declarados marquen una pauta significada. El nodo 1 desarrolla su propia rama, de forma que entre los satisfechos con el barrio se establece también una relación significativa ( $p$-value $\leq 0,0001)$ con la 


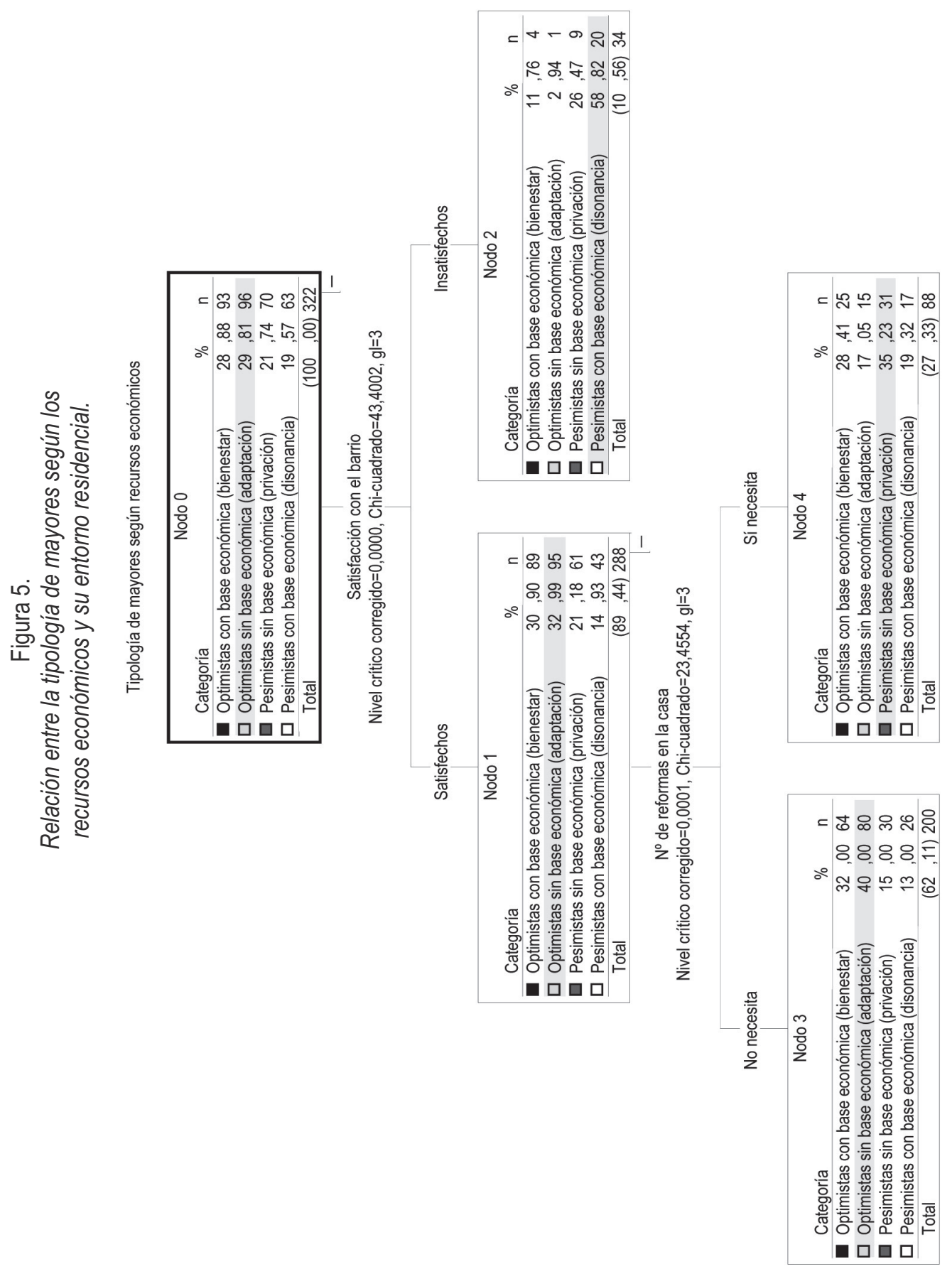


valoración de las condiciones de habitabilidad de la vivienda a través del número de reformas que se entienden como muy necesarias para mantenerla o adaptarla a las circunstancias personales. La mayor parte de los satisfechos con su barrio de residencia considera no necesitar reformas (nodo 3), especialmente aquéllos que mejor valoran sus recursos económicos. Quienes creen que su casa necesita reformas (nodo 4) son, comparativamente, los más "pesimistas", especialmente aquéllos que menos recursos tienen. El indicador sobre calidad global de la vivienda no muestra un nivel estadístico significativo por lo que no ha sido representado en el diagrama.

\section{Conclusiones}

La construcción de índices en Ciencias Sociales requiere la puesta en funcionamiento de estrategias complejas de toma de datos, de adecuación de la información, de utilización de instrumentos específicos y de herramientas analíticas de un cierto grado de complejidad. A partir de la literatura científica, se dispone de las experiencias aportadas por investigadores y de los intereses de las comunidades de científicos sociales que se han decantado desde hace décadas por el análisis de la CdV de los mayores, especialmente la identificación de las dimensiones esenciales que la estructuran, una de las cuales, entre las más destacadas por los propios ancianos, es la económica. La multiplicidad y complejidad de factores (individuales, generacionales, sociales, etc.) que intervienen en el comportamiento de las personas mayores condicionan la adopción de estrategias de investigación también complejas en su diseño e interpretación.

Sin embargo, ahondar en la valoración de la economía de las personas mayores es un empeño muy interesante para establecer su posición en la sociedad y los factores que la explican, al hilo de lo que han perseguido diversas investigaciones. Sin duda es una tarea arriesgada, especialmente cuando se trata de obtener información económica de los individuos y los hogares de personas mayores, condicionada por situaciones que no favorecen la toma de datos adecuados y fiables mediante encuesta. Ésta (i) debe utilizar la "capacidad de recuerdo" de las personas para ofrecer información verídica; (ii) tiene que luchar contra la "negativa social" a hablar de cuestiones económicas ante terceras personas por parte de los mayores, más acentuada que entre la población en general, y (iii) necesita utilizar instrumentos que faciliten la respuesta (umbrales de ingresos), no siempre los más adecuados a efectos analíticos, frente a la solicitud del indicador cantidad de dinero disponible en un periodo determinado. Ninguna solución es definitiva para evitar que los datos de carácter económico solicitados a través de encuestas sean suficientes, fiables y útiles. La más conveniente, sin duda, es aquélla que haga mínimos los efectos de la tasa de no respuesta en las entrevistas, para que los análisis realizados con todas las variables que pueden afectar a la valoración de los recursos económicos sean consistentes con los objetivos previstos.

La investigación social se mueve en un entorno sensible para las personas mayores, 
el de los recursos económicos. En el caso de la Comunidad de Madrid, se trata de personas con ingresos relativamente bajos, en torno a un promedio de $600 €$ mensuales, algo superior a la pensión media de los españoles en el momento de la toma de datos (2005), lo que se relaciona directamente con su nivel de estudios y clase social, de manera que a un menor nivel de estudios y clase social les suelen corresponder unos ingresos también menores. Esta vinculación es consistente con datos objetivos y subjetivos, como el autoposicionamiento económico del hogar detentador de ingresos, siguiendo lógicamente la misma regla: a medida que aumentan los ingresos individuales obtenidos mejora la percepción económica del hogar en el que se reside. No obstante, hay una cierta predisposición de los encuestados a sobrevalorar subjetivamente los ingresos, como se ha demostrado en España y en otros países del entorno sociocultural como Italia (Polverini y Lamura 2005), en la medida en que las personas más mayores y con menor nivel de ingresos tienden a manifestar una cierta satisfacción con los recursos económicos disponibles.

En este juego de realidades (economía disponible) y valoraciones (satisfacción) intervienen no sólo las magnitudes objetivas y subjetivas obtenidas de una encuesta, sino también algunos otros factores condicionados por la idiosincrasia de la persona, la estructura familiar, la historia de su propia vida, las necesidades actuales y las que se prevén para el futuro, los cambios económicos con la edad, los efectos de la acumulación de patrimonio económico o la recepción de herencias (Walker y Mollenkopf 2007).

En sociedades como la madrileña, los mayores son candidatos, en una proporción no desdeñable, a caer en la exclusión social y en la pobreza si no hay adecuación entre recursos y necesidades. Desde un punto de vista subjetivo, la "adaptación" a la situación económica en la que se vive es un mecanismo de supervivencia, muy común entre los mayores, heredado a través de toda su trayectoria vital, como individuos y como miembros de hogares sujetos a decisiones económicas colectivas. A partir de la encuesta CadeViMa, se ha podido comprobar que los que reciben ingresos inferiores a los $300 €$ mensuales, en el entorno de las pensiones no contributivas, reflejan un comportamiento acomodaticio, habituado durante su vida laboral, y a la adecuación de las necesidades a los recursos disponibles así como a las expectativas vitales cubiertas. De la misma manera, aproximadamente el $20 \%$ de las personas mayores encuestadas que han declarado ingresos mensuales elevados manifiesta un cierto nivel de insatisfacción, previsiblemente también ligado con unas necesidades superiores a las habitualmente requeridas a esta edad y heredadas de la etapa adulta, grupo de personas denominado como "disonante".

La encuesta CadeViMa, en su doble enfoque objetivo y subjetivo, fue diseñada para comprobar la consistencia de la relación entre ambas evaluaciones de la dimensión económica de las personas mayores de Madrid (situación económica actual y satisfacción con la misma y sentimiento de seguridad hacia el futuro económico) teniendo como referentes los resultados de investigaciones anteriores, y validar la hipótesis inicial de que el estatus económico de los mayores no se entiende sin enmarcarlo en un conjunto de factores asociados con sus rasgos sociodemográficos y otros elementos de su modo 
y condiciones de vida y sin relacionarlo con el sistema de relaciones personales y sociales de los mayores madrileños. El resultado ha sido la catalogación de los individuos para probar así su aproximación a otras teorías sociales, como la teoría de la calidad de vida basada en la combinación de condiciones objetivas de vida y bienestar subjetivo (Noll 2002). De acuerdo con ello, los mayores han sido clasificados en cuatro tipos, los "optimistas" y los "pesimistas", "con base" y "sin base económica", que cumplen con dos objetivos esenciales de este trabajo: sintetizar de forma significativa (estadística y científicamente) la situación económica medida objetiva y subjetivamente, y utilizar los grupos obtenidos para testar los efectos de otras variables, esenciales en otras dimensiones de la $\mathrm{CdV}$, sobre los recursos económicos.

Como era previsible, las opiniones de carácter económico de los entrevistados están fundamentadas en sus propias condiciones personales, generacionales y en otras facetas de su bienestar personal. Este análisis corrobora el papel destacado que juega la economía de las personas mayores en Madrid, detrás de otros componentes de la CdV como la salud o la familia, y da un papel preponderante a factores como la edad, el nivel educativo o la percepción del estado de salud, determinantes en el momento actual de una situación heredada a partir de unas condiciones creadas y desarrolladas a lo largo de la vida adulta. Los más jóvenes entre los mayores, los que han conseguido una educación que les ha posibilitado un cierto estatus ocupacional y social, y los que manifiestan disfrutar de un mejor estado de salud tienen una valoración positiva y satisfactoria de los ingresos que reciben hoy. La situación contraria se asocia con los más "pesimistas" y que no tienen un nivel de ingresos alto. Aunque la edad y, sobre todo, el sexo no han destacado por su significación estadística en el análisis, se observa la tendencia a un comportamiento diferenciado por género, siendo las mujeres quienes soportan unas condiciones más vulnerables, como consecuencia de su trayectoria social, familiar y laboral en comparación con los hombres y, por lo tanto, con unos recursos económicos más limitados.

Otros aspectos influyen de forma desigual en la valoración de los recursos económicos por parte de los mayores, aunque no de forma separada de las variables anteriormente consideradas. Es el caso de las redes de apoyo social, medidas a través de la necesidad de ayuda para la realización de actividades de la vida diaria. Se ha detectado una relación inversa matizada por el estado de salud como variable que predispone para la ayuda, en el sentido de que un nivel de ingresos bajo tiende a asociarse con una peor percepción del estado de salud y, por lo tanto, con una demanda mayor de ayuda familiar o de otros elementos del entorno social. En una esfera muy cercana a la anterior, entre los que tienen ingresos más elevados, tiende a haber una satisfacción mayor con la forma de vivir, en la medida en que la pareja es la forma más habitual de convivencia, y también se encuentran más satisfechos con las relaciones sociales y con el entorno, contraponiéndose con aquellos mayores que pueden tener una situación más marginal, económica y socialmente, quienes no encuentran tanta satisfacción en la vida que desarrollan. $Y$ en este sentido, la vida en el barrio o localidad de residencia aporta un valor añadido a esta satisfacción personal: si los recursos económicos son suficientes, esta 
satisfacción es más elevada que en el caso contrario, ya que se tienen las condiciones necesarias para asegurar una $\mathrm{CdV}$ relacionada con el entorno residencial más cercano (vivienda y barrio).

Como era previsible, y como también se ha comprobado de forma habitual en los estudios de CdV, la relación entre recursos económicos y las actividades en tiempo libre dan sentido a la vida de las personas mayores. A través de una batería de actividades preguntadas en la encuesta (tiempo personal, de movilidad o de relación) se consigue reflejar la funcionalidad del tiempo disponible, la relación de éstas con los recursos económicos y su utilidad para influir en un comportamiento saludable. En efecto, entre los mayores madrileños, los ingresos económicos predisponen para la realización o no de actividades de ocio, mediante una relación directa, de forma que un menor nivel de ingresos condiciona un ocio pasivo sedentario, mientras que las actividades que suponen un mayor nivel de implicación personal tienden a ser realizadas por personas de estatus económico más alto que los valores medios de los mayores madrileños. Y ello se relaciona directamente con la percepción del estado de salud.

\section{REFERENCIAS BIBLIOGRÁFICAS}

Abellán-García, A., E. del Barrio-Truchado, P. Castejón-Villarejo, C. Esparza-Catalán, G. FernándezMayoralas, L. Pérez-Ortiz, D. Puga-González, F. Rojo-Pérez y M. Sancho-Castiello. 2007. A propósito de las condiciones de vida de las personas mayores. Encuesta 2006. Madrid: Imserso.

Achat, H., I. Kawachi, S. Levine, C. Berkey, E. Coakley y G. Colditz. 1998. "Social Networks, Stress and Health-related Quality of Life." Quality of Life Research 7:735-750.

Ahmed Mohamed, K., F. Rojo-Pérez, G. Fernández-Mayoralas, M. E. Prieto-Flores, V. RodríguezRodríguez y R. Lardiés-Bosque. 2008. "Red familiar y distancia afectiva en la Calidad de Vida de la población mayor." Pp. 375-385 en Envejecimiento, despoblación y territorio. Un análisis sobre la población española, editado por L. López Trigal, A. Abellán García y D. Godenau. León: Universidad de León, Área de Publicaciones.

Alfageme, A. 1999. "La soledad y el género. Una aproximación factorial a la desigualdad económica entre los ancianos españoles de los años noventa". Revista Multidisciplinar de Gerontología 9:147-153.

Alfageme, A. 2001. "Envejecer: una cuestión de desigualdades." Zerbituan 39:38-42.

Andresen, E. M. y D. K. Miller. 2005. "The Future (History) of Socioeconomic Measurement and Implications for Improving Health Outcomes among African Americans." The Journals of Gerontology, Series A: Biological and Medical Sciences 60:1345-1350.

Baxter, J., S. M. Shetterley, C. Eby, L. Mason, C. F. Cortese y R. F. Hamman. 1998. "Social Network Factors Associated with Perceived Quality of Life. The San Luis Valley Health and Aging Study." Journal of Aging and Health 10:87-310.

Bazo, M. T. 1990. La sociedad anciana. Madrid: CIS 113. 
Bazo, M. T. 1999. "Sociología de la vejez." Pp. 47-102 en Envejecimiento y sociedad: una perspectiva internacional, coordinado por M. T. Bazo. Madrid: Médica Panamericana.

Berry, B. 2007. "Does Money Buy Better Health? Unpacking the Income to Health Association after Midlife." Health. An interdisciplinary Journal for the Social Study of Health, IIIness and Medicine 11:199-226.

Bowling, A., Z. Gabriel, D. Banister and S. Sutton. 2002. Adding Quality to Quantity: Older People"s Views on their Quality of Life and its Enhancement. Research Findings: 7. Growing Older Programme 2002. (http://www.shef.ac.uk/Bowling-F7- Consultado el 18 de noviembre de 2010.

Bowling, A. and Z. Gabriel. 2004. "An Integrational Model of Quality of Life in Older Age. Results from the ESRC/MRC HSRC Quality of Life Survey in Britain." Social Indicators Research 69:1-36.

Brown, J., A. Bowling and T. Flynn. 2004. Models of Quality of Life: A Taxonomy, Overview and Systematic Review. London: European Forum on Population Aging Research. (http://www.ageingresearch.group.shef.ac.uk/pdf/qol_review_complete.pdf). Consultado el 18 de noviembre de 2010.

Browne, J. P., C. A. O"Boyle, H. M. McGee, N. J. McDonald y C. R. B. Joyce. 1997. "Development of a Direct Weighting Procedure for Quality of Life Domains." Quality of Life Research 6:301-309.

Bryant, T., D. Raphael, I. Brown, T. Cogan, C. Dallaire, S. Laforest, P. McGowan, L. Richard, L. Thompson y J. Young. 2002. A Nation for All Ages? A Participatory Study of Canadian Seniors" Quality of Life in Seven Municipalities. Toronto: York University, Centre for Health Studies, School of Health Policy and Management. (http://www.utoronto.ca/seniors/seniorsfinalreport.pdf). Consultado el 18 de noviembre de 2010.

Cohn, J., J. A. Sugar. 1991. "Determinants of Quality of Life in Institutions: Perceptions of Frail Older Residents, Staff and Families." Pp. 28-49 en The Concept and Measurement of Quality of Life in the Frail Elderly, coordinated by J. E. Birren, J. E. Lubben, J. C. Rowe y D. E. Deutchman. San Diego, CA: Academic Press.

Demakakos, P., J. Nazroo, E. Breeze y M. Marmot. 2008. "Socioeconomic Status and Health: The Role of Subjective Social Status." Social Science and Medicine 67:330-340.

Diener, E. 1984. "Subjective Well-being." Psychological Bulletin 95:542-575.

Diener, E. y E. Suh. 1997. "Measuring Quality of Life: Economic, Social and Subjective Indicators." Social Indicators Research 40:189-216.

Durán Heras, M. A. y J. Rogero García. 2009. La investigación sobre uso del tiempo. Madrid: CIS, Cuadernos Metodológicos 44.

Evans, G. W., E. Wethington, M. Coleman, M. Worms y E. A. Frongillo. 2008. "Income Health Inequalities Among Older Persons. The Mediating Role of Multiple Risk Exposures." Journal of Aging and Health 20:107-125.

Fernández-Mayoralas Fernández, G., F. Rojo-Pérez, J. M. Rojo-Abuín. 2004. "Components of the Residential Environment and Socio-Demographic Characteristics of the Elderly." Journal of Housing for the Elderly 18:25-49. 
Fernández-Mayoralas, G. y F. Rojo-Pérez. 2005. "Calidad de Vida y salud. Planteamientos conceptuales y métodos de investigación." Territoris 5:117-135.

Fernández-Mayoralas, G., F. Rojo-Pérez, M. E. Prieto-Flores, B. León-Salas, P. Martínez-Martín, M. J. Forjaz, B. Frades-Payo y C. García Izaguirre. 2007. El significado de la salud en la Calidad de Vida de los mayores. Madrid: Portal Mayores, Informes Portal Mayores 74. (http://www.imsersomayores.csic.es/documentos/documentos/fernandezmayoralas-significado-01.pdf). Consultado el 18 de noviembre de 2010.

Friedman, M. I. 1997. Improving the Quality of Life: a Holistic Scientific Strategy. Londres: Praeger.

Gabriel, Z. y A. Bowling. 2004. "Quality of Life in Old Age from the Perspectives of Older People." Pp. 14-34 en Growing Older: Quality of Life in Old Age, editado por A. Walker y C. H. Hennessy. Berkshire: Open University Press.

Gornick, J., T. Munzi, E. Sierminska y T. Smeeding. 2009. "Income, Assets and Poverty: Older Women in Comparative Perspective." Journal of Women, Politics and Policy 30:272-300.

Grundy, E. y G. Holt. 2001. "The Socioeconomic Status of Older Adults: How Should we Measure it in Studies of Health Inequalities?" Journal of Epidemiology and Community Health, 55:895-904.

Grundy, E. y A. Sloggett. 2003. "Health Inequalities in the Older Population: the Role of Personal Capital, Social Resources and Socio-economic Circumstances." Social Science \& Medicine 56:935-947.

Hawins, B. 2005. "Aging Well: Toward a Way of Life for All People." Preventing Chronic Disease 2:1-3.

Hickey, A., C. A. O"Boyle, H. M. McGee and C. R. B. Joyce. 1999. "The Schedule for the Evaluation of Individual Quality of Life." Pp. 119-133 en Individual Quality of Life: Approaches to Conceptualisation and Assessment, edited by C. R. B. Joyce, C. A. O"Boyle y H. M. McGee. Amsterdam: Harwood Academic Publishers.

Higgs, P., M. Hyde, S. Arber, D. Blane, E. Breeze, J. Nazroo y D. Wiggins. 2005. "Dimensions of the Inequalities in Quality of Life in Later Life." Pp. 27-48 en Understanding quality of life in old age, edited by A. Walker. Berkshire: Open University Press.

Instituto de Mayores y Servicios Sociales (Imserso). 2004. Ministerio de Sanidad y Política Social. Encuesta de Condiciones de Vida de los Mayores 2004. Estudio 4597 (http://www.imsersomayores.csic.es/estadisticas/encuestas/index.html).

Instituto de Mayores y Servicios Sociales (Imserso). 2007. Ministerio de Sanidad y Política Social. Informe 2006. Las personas mayores en España. Madrid: Imserso-CSIC.

Instituto de Estadística, Comunidad de Madrid. Padrón Continuo de Habitantes, 2004.

Jang, Y., J. A. Mortimer, W. E. Haley y A. R. Graves. 2004. "The Role of Social Engagement in Life Satisfaction: Its Significance among Older Individuals with Disease and Disability." Journal of Applied Gerontology 3:266-278.

Lamura, G. 2003. European Study of Adult Well-Being: Ageing Well and Material Security in Europe. (http://esaw.bangor.ac.uk//material security final report.pdf. Consultado el 18 de noviembre de 2010. 
Lardiés-Bosque, R., V. Rodríguez-Rodríguez, F. Rojo-Pérez, G. Fernández-Mayoralas, M. E. PrietoFlores y J. M. Rojo-Abuín. 2007. "Significado del ocio y del tiempo libre en la Calidad de Vida de los mayores." Revista Española de Geriatría y Gerontología 40:8-9.

Lawton, M. P. 1991. "Environment and other Determinants of Well-Being in Older People." The Gerontologist 23:349-357.

Li, L. W. 2005. "Predictors of ADL Disability Trajectories Among Low-Income Frail Elders in the Community." Research on Aging 6:615-642.

Mackenbach, J. P. 2006. "Comment. Redistribution of Socioeconomic Resources without a Reduction of Health Inequalities? Some surprises on the road to Utopia." Gaceta Sanitaria 20:178-183.

Meer, J., D. L. Miller, H. S. Rosen. 2003. "Exploring the Health-wealth Nexus." Journal of Health Economics 5:713-730.

Michalos, A. 2003. Essays on the Quality of Life. Dordrecht: Kluwer.

Michael, Y. L., G. A. Colditz, E. Coakley, I. Kawachi. 1999. "Health Behaviors, Social Networks, and Healthy Aging: Cross-sectional Evidence from the Nurses Health Study." Quality of Life Research 8:711-722.

Milligan, C., A. Gatrell y A. Bingley. 2004. "'Cultivating Health": Therapeutic Landscapes and Older People in Northern England." Social Science \& Medicine 9:1781-1793.

Ministerio de Sanidad y Consumo. Encuesta Nacional de Salud de España 2003. (http://www.msc.es/ estdEstudios/estadisticas/encuestaNacioanl/encuestaNac2003/home.htm). Consultado el 18 de noviembre de 2010.

Nguyen, C. T., M-C. Couture, B. Alvarado y M. V. Zunzunegui. 2008. "Life Course Socioeconomic Disadvantage and Cognitive Function Among the Elderly Population of Seven Capitals in Latin America and the Caribbean." Journal of Aging Health 20:347-362.

Noll, H-H. 2002. "Towards a European System of Social Indicators: Theoretical Framework and System Architecture." Pp. 47-87 en Assessing Quality of Life and Living Conditions to Guide National Policy, coordinated by M. R. Hagerty, J. Vogel y V. Moller. Dordrecht: Kluwer.

Oswald, F., H. Wahl, H. Mollenkopf, O. Schilling, D. Neumann and K. Schakib-Ekbatan. 2005. ENABLE AGE Survey Study T1. Preliminary Descriptive Findings. Summary of German National Report WP3. (http://www.enableage.arb.lu.se/documents/Comparative Report M3 M6.pdf). Consultado el 18 de noviembre de 2010.

O'Boyle, C. A., H. M. McGee, A. Hickey, C. R. B. Joyce, J. Browne, K. O'Malley and B. Hiltbrunner. 1993. The Schedule for the Evaluation of Individual Quality of Life. User manual. Dublin: Royal College of Surgeons in Ireland.

Park, S., S. Jang and D. Kim. 2010. "Gender Differences as Factors in Succesful Aging: a Focus on Socioeconomic Status." Journal of Biosocial Sciences 42:99-111.

Pérez Ortiz, L. 1997. Las necesidades de las personas mayores. Vejez, economía y sociedad. Madrid: Imserso. 
Pérez Ortiz, L. 2006. La estructura social de la vejez en España. Nuevas y viejas formas de envejecer. Madrid: Imserso.

Pinquart, M. and S. Sorensen. 2000. "Influences of Socioeconomic Status, Social Network, and Competence on Subjective Well-being in Later Life: A Meta-analysis." Psychology and Aging 2:187-224.

Polverini, F. and G Lamura. 2005. "Italy: Quality of Life in Old Age." Pp. 179-200 en Growing Older in Europe, edited by A. Walker. Berkshire: Open University Press.

Regidor, E., D. Martínez, P. Astasio, P. Ortega, M. E. Calle y D. Domínguez. 2006. "Evolución de las desigualdades socioeconómicas y las desigualdades en la percepción de la salud en España." Gaceta Sanitaria 20:1-5.

Rojo-Pérez, F., G. Fernández-Mayoralas y E. Pozo-Rivera. 2000. "Envejecer en casa: los predictores de la satisfacción con la casa, el barrio y el vecindario como componentes de la Calidad de Vida de los mayores en Madrid." Revista Multidisciplinar de Gerontología 4:222-233.

Rojo-Pérez, F., G. Fernandez-Mayoralas, V. Rodriguez-Rodríguez, J. M. Rojo-Abuín. 2007. "The Environments of Ageing in the Context of the Global Quality of Life among Older People Living in Family Housing." Pp. 123-150 en Quality of Life in Old Age, coordinated by H. Mollenkopf and A. Walker. Dordrecht: Springer.

Rojo-Pérez, F., C. Delgado-Sanz, G. Fernández-Mayoralas, M. J. Forjaz, K. Ahmed-Mohamed, P. Martínez-Martín, M. E. Prieto-Flores y J. M. Rojo-Abuín. 2009a. "Informal Support According to Level of Competence Related to Health and Functioning in Quality of Later Life." Pp. 64-85 en Quality of Life Improvement through Social Cohesion, edited by W. Ostasiewicz. Wroclaw: Publishing House of Wroclaw University of Economics.

Rojo-Pérez, F., G. Fernández-Mayoralas, M. J. Forjaz, C. Delgado-Sanz, K. Ahmed Mohamed, P. Martínez-Martín, M. E. Prieto-Flores y J. M. Rojo-Abuín. 2009b. Población mayor, Calidad de Vida y redes de apoyo: demanda y prestación de cuidados en el seno familiar. Madrid: Escuela Nacional de Sanidad, Instituto de Salud Carlos III, Ministerio de Ciencia e Innovación. (http://www.isciii.es/ htdocs/publicaciones/documentos/Poblacion_mayor.pdf). Consultado el 18 de noviembre de 2010.

Sánchez Vera, P. 2000. "Sociología de la vejez versus economía de la vejez." Papers 61:39-88.

Scharfs, Th., Ch. Phillips, P. Kinsgton and A. Smith. 2000. "Social Exclusion and Older People: towards a Conceptual Framework." Working Paper 6. Centre for Social Gerontology (http://www.keele. ac.uk/research/lcs/csg/downloads/social exclusion.pdf). Keele University, Centre for Social Gerontology, Working Paper no.6, 29 pags. Consultado el 18 de noviembre de 2010

Schyns, P. 1998. "Cross-national Differences on Happiness: Economic and Cultural Factors Explored." Social Indicators Research 43:3-26.

Sirgy, M. J. 2001. Handbook of Quality-of-life Research: an Ethical Marketing Perspective. Dordrecht: Kluwer.

Smith, A. 2000. "Researching Quality of Life of Older people: Concepts, Measures and Findings." Working Paper 7. Keele University, Centre for Social Gerontology, Working Paper no.7, 29 pags. (http:// www.keele.ac.uk/research/lcs/csg/downloads/research_quality.pdf). Consultado el 18 de noviembre de 2010 
Smith, J. P. and R. S. Kinsgton. 1997. "Race, Socioeconomic Status, and Health Late in Life." Pp. en Racial and Ethnic Differences in the Health of Older Americans, coordinated by L. G. Martin y B. Soldo. Washington, D. C.: National Academy Press.

Taylor, S. E. and T. E. Seeman. 1999. "Psychosocial Resources and the SES-Health Relationship." Annals of the New York Academy of Sciences 890: 210-225.

Trinidad, A. 2008. "Estrategias sociales y económicas de los nuevos jubilados." Revista Española de Investigaciones Sociológicas 115:135-163.

Tsimbos, C. 2010. "An Assessment of Socio-economic Inequalities in Health among Elderly in Greece, Italy and Spain." International Journal of Public Health 55:5-15.

Walker, A. 2005. Understanding Quality of Life in Old Age. Berkshire: Open University Press.

Walker, A. and H. Mollenkopf. 2007. "International and Multidisciplinary Perspectives on Quality of Life in Old Age." Pp. 3-13 en Quality of Life in Old Age, coordinated by H. Mollenkopf and A. Walker. Dordrecht: Springer.

Walker, A. and C. H. Hennessy, eds. 2004. Growing Older: Quality of Life in Old Age. Berkshire: Open University Press.

Warr, P. and V. Butcher, I. Robertson. 2004. "Activity and Psychological Well-being in Older People." Aging \& Mental Health 2:172-183.

Weidekamp-Maicher, M. and G. Naegele. 2007. "Economic Resources and Subjective Well-being in Old Age." Pp. 65-84 en Quality of Life in Old Age, coordinated by H. Mollenkopf and A. Walker. Dordrecht: Springer. 
VICENTE RODRÍGUEZ RODRÍGUEZ es Doctor en Geografía e Historia y Profesor de Investigación en el CCHS (CSIC). Dentro del Grupo de Investigación en Envejecimiento (GIE-CSIC) participa en el análisis de la movilidad residencial de las personas mayores, y sus factores asociados. Así mismo está implicado en el estudio longitudinal del envejecimiento en España (proyecto ELES).

FERMINA ROJO-PÉREZ es Doctora en Geografía e Investigadora en el CCHS (CSIC), centra su interés en la calidad de vida en la vejez, su multidimensionalidad y factores explicativos. Como miembro del GIE-CSIC y de la UA INGEMA-CSIC, está involucrada en el estudio interdisciplinar con diseño longitudinal del envejecimiento en España (proyecto ELES).

GLORIA FERNÁNDEZ-MAYORALAS es Doctora en Geografía e Investigadora en el CCHS-CSIC, centra su interés en las condiciones de salud y del entorno físico y social que promueven la calidad de vida global en la vejez. Desde 1989 integra el GIE-CSIC y, en la actualidad, forma parte de la UA INGEMA-CSIC. Desde esta última colabora en el Estudio Longitudinal del Envejecimiento en España (ELES).

KARIM AHMED MOHAMED es Diplomado en Trabajo Social (2004) y Licenciado en Ciencias Políticas (2002), Antropología (2006) y Sociología (2007). Actualmente es becario FPI en el Centro de Ciencias Humanas y Sociales (CSIC). Sus principales intereses de investigación son: envejecimiento, calidad de vida, asociacionismo y trabajo social comunitario.

RAÚL LARDIÉS BOSQUE es Doctor en Geografía y Profesor Ayudante Doctor en el Departamento de Geografía y Ordenación del Territorio de la Universidad de Zaragoza. Ha investigado en los campos del desarrollo rural y las migraciones, y sobre el envejecimiento, la movilidad residencial y la calidad de vida de la población mayor.

MARÍA-EUGENIA PRIETO-FLORES es Doctora en Geografía e Investigadora Postdoctoral en el Instituto de Salud Carlos III. Pertenece a un equipo multidisciplinar en calidad de vida y envejecimiento. Su actividad investigadora se enmarca en la geografía de la salud y la calidad de vida en la vejez, con una aproximación multimétodo.

JOSÉ-MANUEL ROJO-ABUÍN es Diplomado y licenciado en Ciencias y Técnicas Estadísticas (Universidad Complutense de Madrid), es responsable de la Unidad de Análisis Estadístico en el CCHS (CSIC), donde participa en el diseño y análisis estadísticos de las investigaciones realizadas en el centro. Es miembro de la Sociedad Española de Estadística e Investigación Operativa.

RECIBIDO: 26/11/2008

ACEPTADO: $24 / 05 / 2010$

Publicado on-line: 02/12/2010 